\title{
Genome-Wide Identification of Tea Plant bHLH Transcription Factor Family and Discovery of Candidate Regulator for Trichome Formation
}

\section{Renjian Liu}

South China Agricultural University

\section{Yuyuan Wang}

South China Agricultural University

\section{Song Tang}

South China Agricultural University

Jiarong Cai

South China Agricultural University

Shaoqun Liu

South China Agricultural University

Peng Zheng ( $\square$ zhengp@scau.edu.cn )

South China Agricultural University

\section{Binmei Sun}

South China Agricultural University

\section{Research Article}

Keywords: tea plant, trichome formation, bHLH, expression pattern, transcriptional activation

Posted Date: January 25th, 2021

DOl: https://doi.org/10.21203/rs.3.rs-148784/v1

License: (c) (i) This work is licensed under a Creative Commons Attribution 4.0 International License. Read Full License 


\title{
Genome-wide identification of tea plant bHLH transcription factor family and discovery of candidate regulator for trichome formation
}

\author{
Renjian Liu ${ }^{1}$, Yuyuan Wang ${ }^{1}$, Song Tang ${ }^{1}$, Jiarong Cai ${ }^{1}$, Shaoqun Liu ${ }^{1}$, Peng Zheng ${ }^{1 *}$, Binmei Sun ${ }^{1 *}$ \\ liurj_scau@163.com; wangyy@stu.scau.edu.cn; tegantang@163.com; caijr@stu.scau.edu.cn; scauok@scau.edu.cn; \\ *Correspondence: zhengp@scau.edu.cn; binmei@scau.edu.cn \\ 1. College of Horticulture, South China Agricultural University, Guangzhou, Guangdong 510642, People's Republic of China;
}

Abstract: Leaf trichomes play vital roles in plant resistance and tea quality. Basic helix-loop-helix (bHLH) transcription factors (TFs) play an important role in regulating plant development and growth. However, it is poorly understood whether bHLH TFs are associated with trichome formation in tea plant. In this study, a total of $134 \mathrm{CsbHLH}$ proteins were identified in the Camellia sinensis var. sinensis (CSS) genome. All identified proteins were divided into 19 subgroups according to the Arabidopsis thaliana classification. Phylogenetic tree analysis indicated that the members of group IIIc-I and group IIIc-II might be associated with trichome formation. Expression analysis showed that the candidate genes associated with trichome formation in tea plant were primarily located in cluster 1, cluster 3 and cluster 5. The expression patterns of CsbHLH116, CsbHLH133, CsbHLH060, CsbHLH028, CsbHLH024, CsbHLH112 and CsbHLH053 from these clusters were similar to the trichome distribution in tea plants. Notably, CsbHLH024 and CsbHLH133 were highly expressed in the young tissues of different cultivars. CsbHLH024 and CsbHLH133 possessed transcriptional activation ability and could interact with CsTTG1, a regulator of tea trichome formation. This study provides useful information for further research on the function of CsbHLHs in the regulation of trichome formation.

Keywords: tea plant; trichome formation; bHLH; expression pattern; transcriptional activation;

\section{Introduction}

Trichomes developed from epidermal cells are mainly distributed on the undersurface of plant leaves [1-4]. Plant leaf trichomes are as an important basis for botanical classification and play key roles in plant resistance to biotic and abiotic stress. According to the morphology and function of trichomes, they are classified into nonbranched and branched trichomes or nonglandular and glandular trichomes [5-8]. Glandular trichomes protect plants from herbivores and insects by accumulating and secreting a series of secondary metabolites, such as alkaloids, nicotine and terpenes $[9,10]$. Nonglandular trichomes can enhance tolerance to extreme temperature, drought and ultraviolet radiation [1114]. Trichome formation has been systematically investigated in Arabidopsis thaliana, Solanum lycopersicum, Cucumis sativus L., Oryza sativa L., Nicotiana tabacum L., Gossypium spp. and Glycine max [15-21]. Additionally, trichome formation is induced by cell differentiation. Arabidopsis thaliana trichome development is an ideal model for the study of cell differentiation [22].

Tea, one of the three major nonalcoholic beverages, possesses high nutritional and health-benefitting properties $[23,24]$. Tender leaves are the main raw material used for tea production. The apical buds and young leaves are attached to trichomes in most tea cultivars; thus, leaf trichomes have become a critical diagnostic characteristic in tea taxonomy. Additionally, leaf trichomes are essential for the appearance and quality of tea products. Plentiful trichomes are generally considered to indicate high quality in Chinese tea. Tea trichomes are abundant in metabolites including theanine, catechins, volatiles and caffeine $[25,26]$. They possess especially high contents of benzoic acid derivatives, lipid oxidation derivatives and monoterpene derivatives, which contribute to tea flavor and aroma [27]. Some signaling genes related to diseases, antiherbivore effects and antiabiotic peptides have been shown to be specifically transcribed in tea trichomes [27]. Therefore, trichomes play an important role in tea plant.

The basic helix-loop-helix (bHLH) transcription factors (TFs) constitute the second largest TF family in plants [28]. Their conserved domains are composed of 50-60 amino acids and include two different functional regions, a basic region 
and a helix-loop-helix (HLH) region [29-31]. The basic region is located in the N-terminal domain; it consists of 13-17 amino acids and binds to the consensus hexanucleotide E-box (CANNTG). The HLH region in the C-terminal domain includes approximately 40 amino acids and contributes to the formation of homodimeric complexes and heterodimeric complexes as well as the promotion of interactions with other TFs [32-35]. bHLH TFs play important roles in responses to stresses, secondary metabolism biosynthesis and plant growth and development [29,36-39]. Numerous studies have demonstrated that bHLH TFs play a critical role in trichome formation. The bHLH proteins GLABRA3 (GL3) and ENHANCER OF GLABRA3 (EGL3) are important for the regulation of trichome formation in Arabidopsis thaliana $[40,41]$. GL3 and EGL3 induce trichome formation by targeting GLABRA2 (GL2) transcription by interacting with the WD40 and R2-R3 MYB proteins [42-44]. GL3 also facilitates trichome branching formation by positively regulating FPR4 expression [45]. Whereas trichome formation in tomato is independent of SIGL3 [46]. Trichome formation is extremely complex. Whether bHLH TFs are related to trichome formation in tea plant is less well understood.

In this study, the bHLH family was characterized in Camellia sinensis var. sinensis (CSS) genome and systematically carried out characteristic analysis to understand the biological function of candidate bHLH TFs in the regulation of trichome formation in tea plant. The potential tea trichome regulatory network between candidate bHLHs and other TFs was predicted. Thus, this study provides useful information for further research on the function of CsbHLH TFs in the regulation of trichome formation.

\section{Materials and Methods}

\section{Identification of the $b H L H$ gene family in tea plant}

The bHLH TF sequences were acquired from the TPIA (http://tpia.teaplant.org) [26]. The SMART database [47], National Center for Biotechnology Information (NCBI) conserved domain search service [48] and the hidden Markov model (HMM) profile of the bHLH domain (PF00010) in the Pfam database [49] were used to filter redundant bHLH proteins in tea plant. The full-length amino acid sequences, MW, theoretical pI and instability index of these proteins were predicted using the ExPASy server (https://web.expasy.org/protscale). The CsbHLHs were renamed CsbHLH001 to CsbHLH134 based on the gene ID order.

\section{Conserved motif and gene structure characterization}

The conserved motifs of the bHLH protein were identified using MEME (http://meme.nbcr.net/meme/cgibin/meme.cgi) with the following parameter settings: site distribution, any number of repetitions; number of motifs, 15; maximum motif width, 100; minimum motif width, 6; maximum number of sites, 100; minimum number of sites, 5 [50]. The gene structure, including both the coding DNA sequences (CDSs) and genomic sequences of bHLHs was determined with TBtools software [51].

\section{Phylogenetic tree analysis}

The bHLH protein sequences of Arabidopsis thaliana (128) were downloaded from The Arabidopsis Information Resource (TAIR) database (https://www.arabidopsis.org/), and those of Oryza sativa L. (144) and Actinidia chinensis (164) were acquired from the Plant TF Database version 4.0 [52]. All of them were renamed and listed in Table S4. Moreover, the conserved bHLH domains of these proteins were subjected to multiple alignment analysis using ClustalX 2.1 (http://www.clustal.org/) with the default parameters. MEGA X was used to construct neighbor-joining phylogenetic trees with 1000 bootstrap replications [53], and the phylogenetic trees were optimized using Evolview v3 [54].

\section{Transcriptome data analysis}

The transcriptome data of tea plant $b H L H s$ were obtained from TPIA [26]. The expression patterns of CsbHLHs in different developing leaf tissues, including the apical buds and young, mature leaf and old leaves, were determined with R Language software. 


\section{Plant material}

'Renhuabaihao'('RHBH'), 'Yinghongjiuhao' ('YH9') and 'Baiyedancong' ('BYDC') were cultivated at South China Agricultural University (Guangzhou, China). According to institutional, national and international guidelines, the material used for research purposes does not require specific permissions. The apical bud, young leaf, mature leaf and old leaf tissues of three tea plant cultivars were collected and used to conduct trichome observations by stereoscopy (Carl Zeiss, Germany).

\section{qRT-PCR analysis}

The total RNA of the tea plant samples was extracted and isolated using the HiPure Total RNA kit (R4111, Magen, China). RNA reverse transcription was carried out with the HiScript III RT SuperMix for qPCR Reagent Kit with gDNA Wiper (R323-01, Vazyme, China). The primers employed for qPCR were designed with the NCBI Primer design tool (https://www.ncbi.nlm.nih.gov/tools/primer-blast/index.cgi?LINK_LOC=BlastHome). All primers are listed in Table S5. Ubi and SAND1 were used as the reference genes. qRT-PCR analysis was performed with the Bio-Rad CFX384 Touch TM system (Bio-Rad, Hercules, CA, USA) with routine procedures [55]. Relative expression was calculated using the $2^{-\Delta \Delta C t}$ method [56]. All the samples were analyzed with three biological replicates and three technical replicates for each biological replicate.

\section{Subcellular localization}

The coding sequences of CsbHLH133 and CsbHLH024 without the termination codon were cloned into a pEAQEGFP vector. The recombinant plasmids and nuclear localization signal (NLS-DsRed) were transformed into Agrobacterium tumefaciens strain GV3101, which was mixed and injected into tobacco (Nicotiana benthamiana) leaves. After 48 hours, the tobacco leaves were collected for fluorescence microscopy observations (Carl Zeiss, Germany).

\section{Dual-luciferase reporter assay}

A dual-luciferase reporter assay system (Promega, USA) was used for transcriptional activation. The full-length coding sequences of CsbHLH133 and CsbHLH024 were ligated into the pEAQ-PBD vector fused with the GAL4 DNAbinding domain under the control of the CaMV 35S promoter. The empty vector, the reporter gene (GAL-LUC) and the generated constructs were transformed into the GV3101 Agrobacterium strain. The Agrobacterium strain containing the empty vector or the constructs and the reporter were coinfiltrated into tobacco leaves. After three days, the tobacco leaves were collected, and the activity of the Renilla LUC/firefly was measured according to a previously described protocol [39].

\section{The construction of potential regulatory network}

The protein sequences of TFs associated with Arabidopsis thaliana trichome formation, including MYB23, TRY, GL3, ETC1, GL1, GL2, CPC, TTG1, ETC2, SAD2 and EGL3 [57] were obtained from the TAIR database (https://www.arabidopsis.org/). They were submitted to the STRING server (https://string-db.org) for protein-protein interaction network functional enrichment analysis with the default parameters.

\section{Yeast two-hybrid assay}

The full-length cDNAs of CsbHLH024, CsbHLH133 and CsTTG1 were cloned into the pGBKT7 and pGADT7 vectors, respectively, via one-step cloning (C112, Vazyme, China). The resulting positive, negative and recombinant plasmids were subsequently transformed into a Y2HGold yeast strain (YC1002, Weidi Biotechnology, China). A yeast two-hybrid assay was then performed according to manufacturer's instructions (Clontech), and image acquisition was performed via Adobe Illustrator CS2020 (Germany, Zeiss). All the primers used are listed in Table S5. 


\section{Identification and conserved domain analysis of CsbHLHs}

A total of 134 CsbHLHs were identified in the Camellia sinensis var. sinensis (CSS) genome [26]. The physical and chemical properties of the 134 CsbHLH proteins were predicted. As shown in Table S1, all of the identified proteins encoded 146-1038 amino acids. Their molecular weights (MWs) and the theoretical isoelectric points (pI) ranged from 16.36 kDa to $114.15 \mathrm{kDa}$ and 4.72 to 9.67, respectively. Only four CsbHLHs were stable (instability index<40). The spliced sequences in the CCS genome were not clustered on any chromosomes [26], and the 134 CsbHLHs were renamed CsbHLH001 to CsbHLH134 based on the gene ID order (Table S1).

The conserved domains of the CsbHLH proteins were determined via multiple alignment analysis. As shown in Figure 1, the bHLH domains possessed four conserved regions: basic, first helix, loop and second helix regions. More than $50 \%$ of the residues in the bHLH domain were conserved: six residues (His-5, Ala-8, Glu-9, Arg-10, Arg-12 and Arg-13) in the basic region; four residues (Asn-17, Arg-19, Leu-23 and Pro-28) in the helix 1 region; two residues (Lys32 and Asp-34) in the loop region; and nine residues (Lys-35, Ala-36, Ser-37, Leu-39, Ala-42, Ile-43, Tyr-45, Lys-47 and Leu-49) in the helix 2 region. Arg-12, Arg-13, Leu-23 and Leu-49 were highly conserved (Figure 1). Additionally, the basic region was absent in CsbHLH067, while the loop and helix 2 regions were not present in CsbHLH056 (Figure S1).

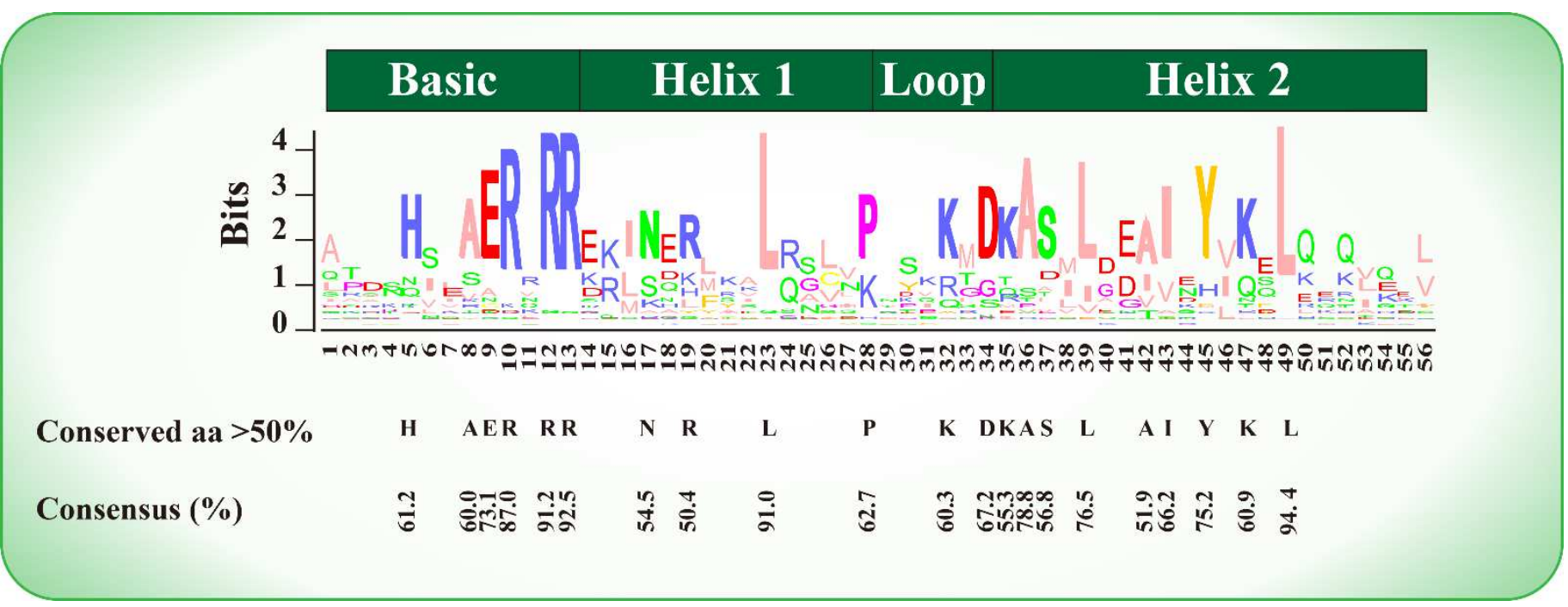

Figure 1. Conserved residues analysis of bHLH domains. The height of each residue indicates the conservation rate. The black letters represent the residues with a consensus ratio greater than $50 \%$.

\section{Phylogenetic tree analysis of CsbHLH proteins}

A neighbor-joining phylogenetic tree including all bHLH proteins identified in tea plant and those from Arabidopsis thaliana was constructed for the classification of CsbHLH proteins. The CsbHLH proteins were divided into 19 subgroups according to the classification in Arabidopsis thaliana [58] (Figure 2). Group IIIc was subdivided into group IIIc-I and group IIIc-II. The numbers of AtbHLHs and CsbHLHs in each group are listed in Table S2. The members of group II included one CsbHLH and four AtbHLHs. Group XII contained the largest numbers of CsbHLHs (21) and AtbHLHs (16). The difference between the members of the CsbHLHs and AtbHLHs within the same group might be result from the unequal duplication of the bHLH family during species differentiation. Group VI and group X contained no CsbHLH members, while group XIII included only CsbHLH members. To clarify whether group XIII was specific to tea plant, all the bHLH proteins of tea plant were used to construct a neighbor-joining phylogenetic tree along with those of Arabidopsis thaliana, Oryza sativa L. and Actinidia chinensis (Figure S2). The bHLHs in group XIII included bHLHs from Oryza sativa L. and Actinidia chinensis. Therefore, group XIII was not specific to tea plant. 
The TFs classified in the same group in the phylogenetic tree might possess similar functions. As shown in Figure 2, some Arabidopsis thaliana bHLH TFs related to trichome formation were identified, including AtTT8 [59], AtEGL3 [60], AtGL3 [61], and AtMYC1 [62], which were mapped to AtbHLH042, AtbHLH001, AtbHLH002 and AtbHLH012, respectively. All of these TFs were included in groups IIIc-I and IIIc-II. Therefore, group IIIc-I and group IIIc-II were defined as 'trichome-related groups', which might be involved in tea trichome formation.

In addition, the conserved motifs and gene structures of the CsbHLHs were analyzed. Information on the 15 identified motifs is listed in Table S3. The results showed that members of the same group might possess similar motifs and gene structures (Figure S3).

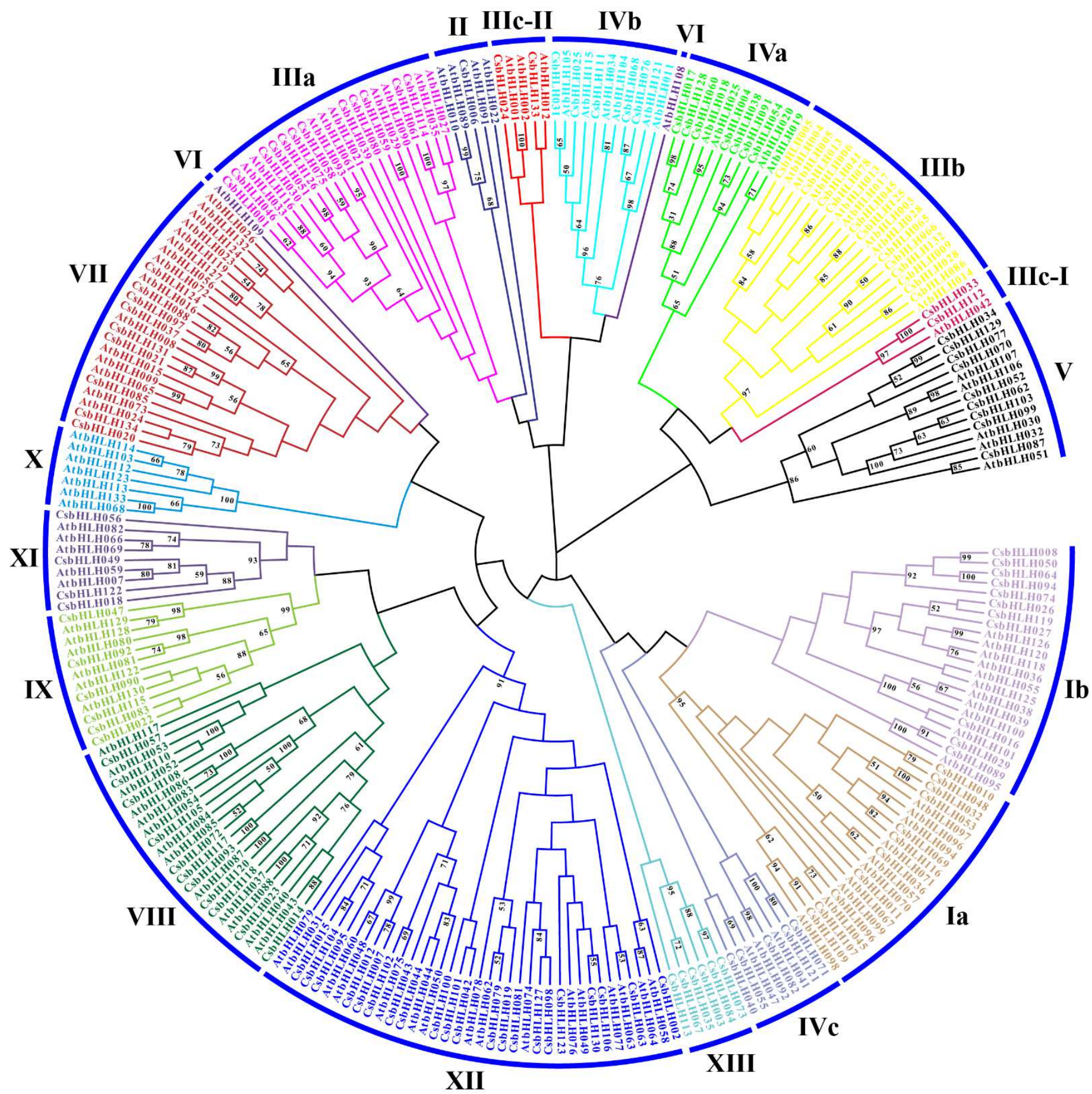

Figure 2. Phylogenetic tree analysis of tea plant and Arabidopsis thaliana bHLH proteins. Different colored branches indicate different subgroups. 


\section{Transcriptome analysis of CsbHLHs in different developing leaf tissues in tea plant}

Trichomes are important for tea plant and directly affect the quality and flavor of tea. Leaf trichomes are distributed mainly in the apical buds and the young leaves. To further understand the potential function of CsbHLH proteins during leaf trichome formation in tea plant, the expression patterns of CsbHLHs in different developing leaf tissues were determined, including apical buds, young leaves, mature leaves and old leaves. The RNA-seq data of CsbHLHs in different developing leaf tissues were downloaded from the Tea Plant Information Archive (TPIA). Eight CsbHLHs (CsbHLH029, CsbHLH059, CsbHLH062, CsbHLH066, CsbHLH089, CsbHLH102, CsbHLH110 and CsbHLH117) were transcribed at low levels in different developing leaf tissues and could not be quantified. According to the similarity of the observed expression patterns, a heatmap showed hierarchical clustering into 10 clusters (Figure 3). The expression patterns of CsbHLHs in cluster 1, cluster 3 and cluster 5 were consistent with the distribution of tea leaf trichomes and were primarily associated with the apical buds and the young leaves. CsbHLHs in cluster 2, cluster 4, cluster 6, cluster 7 and cluster 9 were highly expressed in the mature and old leaves of tea plant, while the expression of CsbHLHs in cluster 8 and cluster 10 was high in the apical buds and the old leaves. These results indicated that the members of cluster 1, cluster 3 and cluster 5 might be involved in trichome formation in tea plant.

Trichome formation is closely related to root hair formation in plant. In Arabidopsis thaliana, a MYB-bHLH-WD40 (MBW) transcriptional activator complex can promote trichome formation and inhibite the root hair formation by inducing the GL2 expression [42]. Therefore, the expression profiles of CsbHLHs of cluster 1, cluster 3 and cluster 5 in different tissues including the apical buds, young leaves, root, flower, fruit and stem were investigated. The RNA-seq data of CsbHLHs of cluster 1, cluster 3 and cluster 5 in different tissues were downloaded from the TPIA. The heat map showed that the gene with the high expression in the apical buds and young leaves possessed low expression in the root of tea plant (Figure S4). 


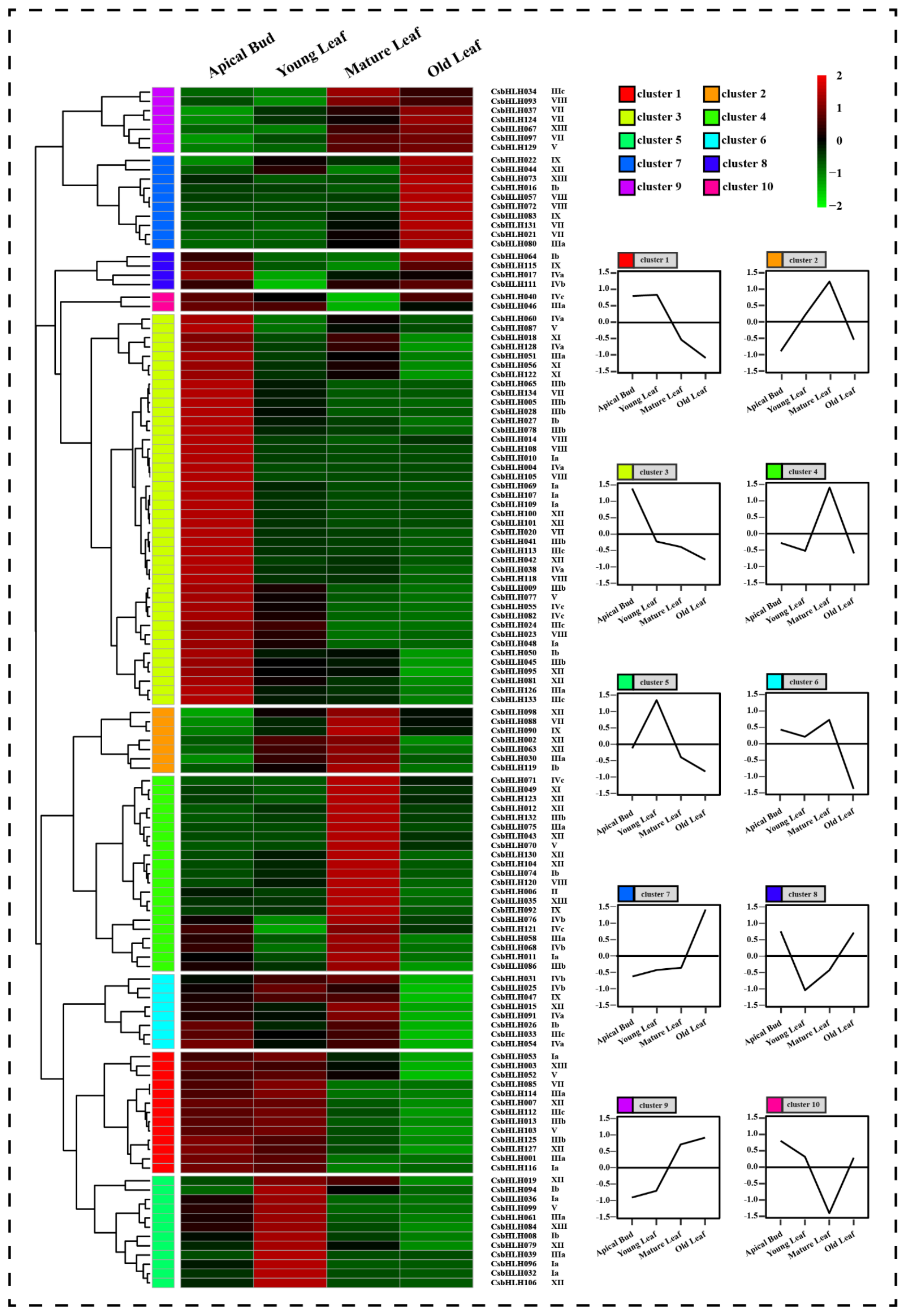


Figure 3. Transcriptome analysis of CsbHLHs in different developing leaf tissues. The name of each gene and the short name of the phylogenetic group are listed on the right of the heatmap. Line charts were generated using the mean value for the whole cluster.

\section{Expression patterns of $\mathrm{Cs} b \mathrm{HLH}$ in different developing leaf tissues in tea plant}

To verify the expression pattern of $\mathrm{CsbHLHs}$ in different developing leaf tissues, twenty CsbHLHs were analyzed using qRT-PCR. The expression of CsbHLH116, CsbHLH033, CsbHLH133, CsbHLH060, CsbHLH028 and CsbHLH040 was upregulated in apical bud tissue, while the expression of CsbHLH024, CsbHLH112, CsbHLH119, CsbHLH002 and CsbHLH053 exhibited a peak in young leaf tissue (Figure 4). The expression of these genes decreased with leaf maturation, similar to what was observed for the distribution of tea trichomes. The comprehensive and systematic analysis of the topology of the phylogenetic tree and the expression patterns indicated that CsHLH024 and CsbHLH133 were likely candidates for the regulation of trichome formation in tea plant.
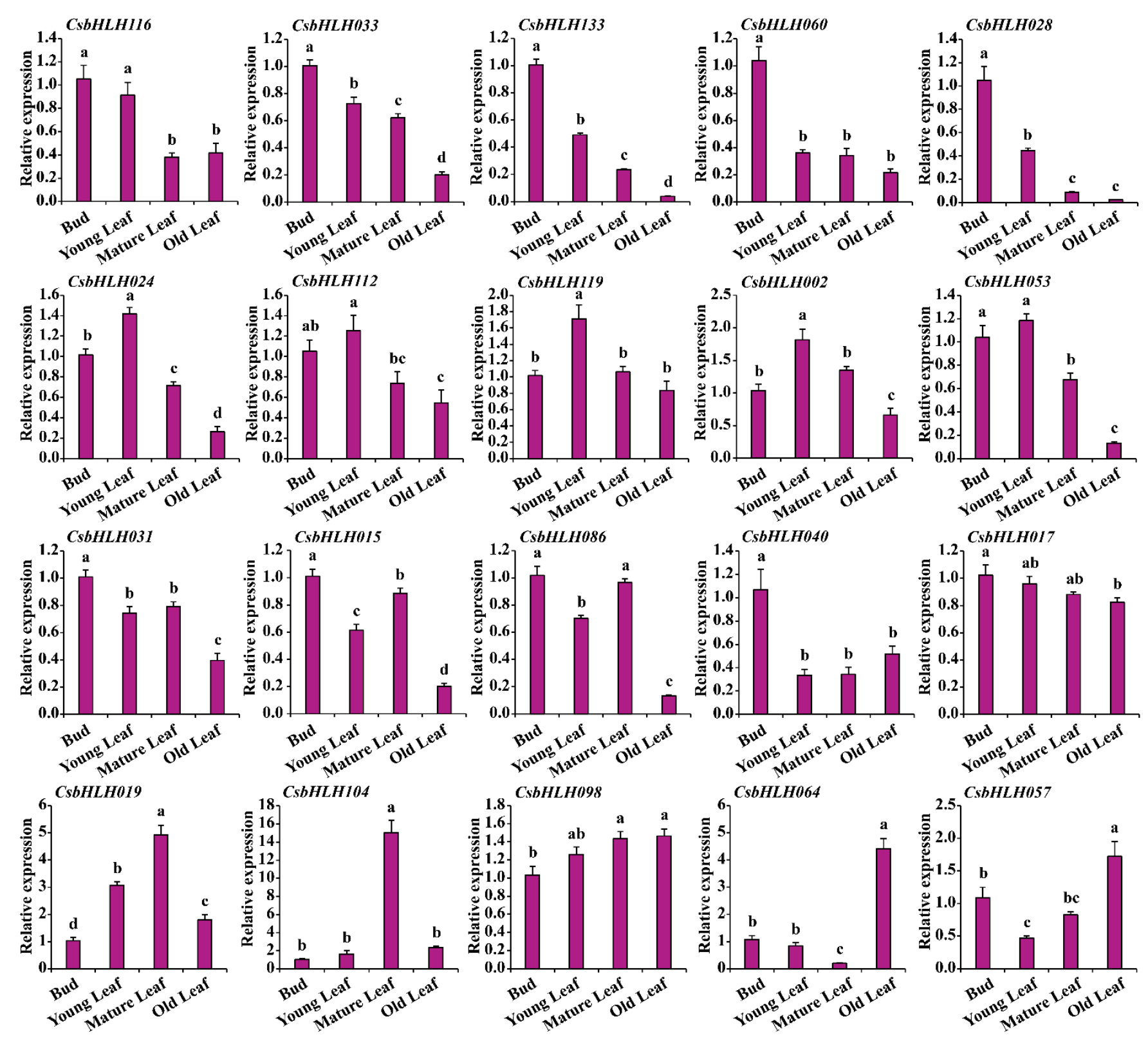
Figure 4. The expression patterns of $\mathrm{Cs} b H L H s$ in different developing leaf tissues. The different letters in the figures indicate significantly different values $(\mathrm{P}<0.05$, Tukey's test). For all developing leaf tissues, three biological replicates and three technical replicates of each biological replicate were performed.

B

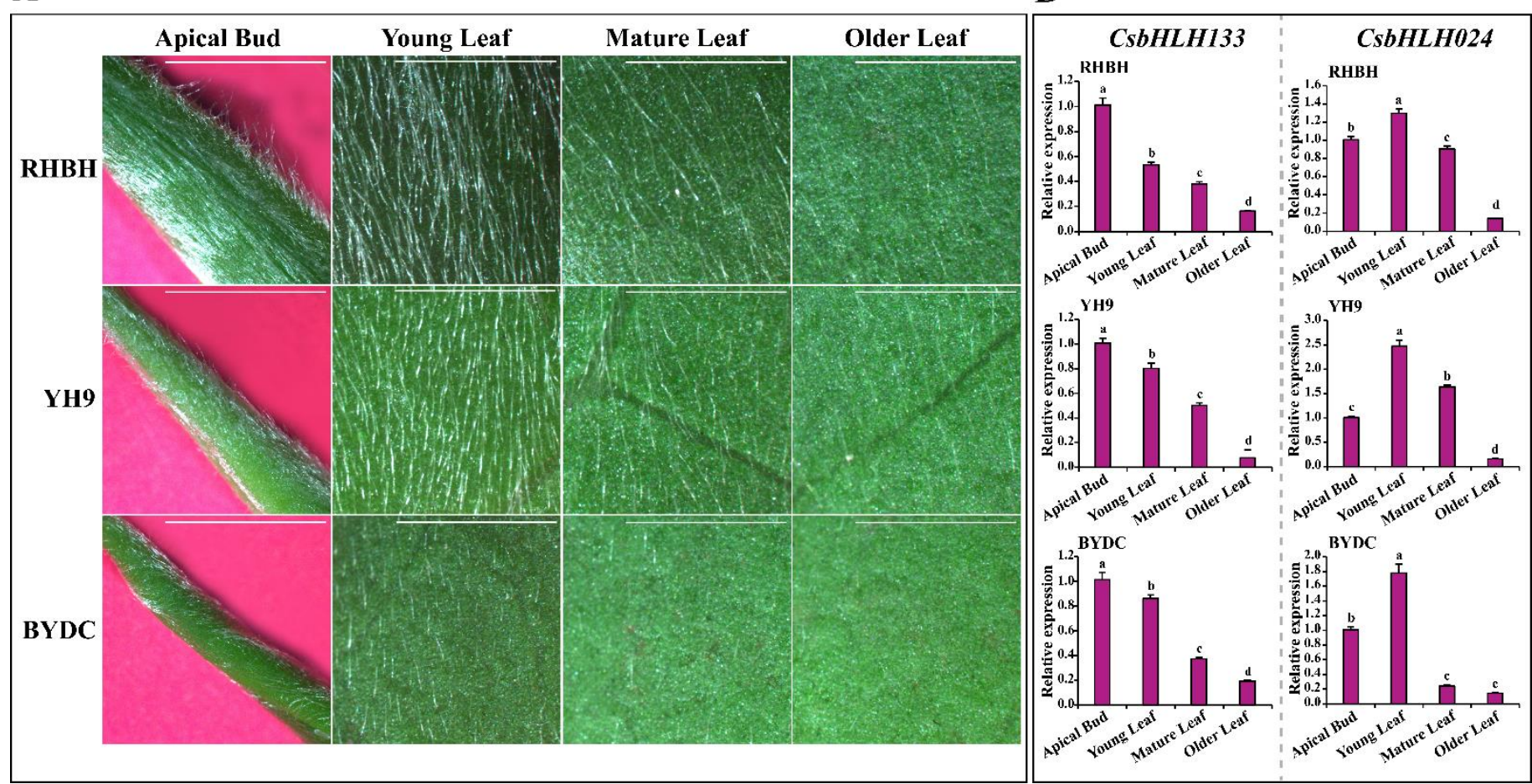

Figure 5. The trichome distribution and the expression patterns of CsbHLH024 and CsbHLH133 in different tea plant cultivars. A. The trichome distribution in cultivars 'RHBH', 'YH9' and 'BYDC'. B. The expression patterns of CsbHLH024 and CsbHLH133 in different developing leaf tissues of different tea plant cultivars. The different letters in the figures indicate significantly different values $(\mathrm{P}<0.05$, Tukey's test).

\section{CsbHLH133 and CsbHLH024 act as transcriptional activators}

To verify whether CsbHLH133 and CsbHLH024 have transcriptional activation ability, Subcellular location and transcriptional activation assays were performed. As shown in Figure 6A, fluorescence signals from the empty vector were located in the cell nucleus and cytoplasm, while those of the 35S: CsbHLH133-GFP and 35S: CsbHLH024-GFP proteins were only found in the cell nucleus. These results indicated that CsbHLH133 and CsbHLH024 were localized in the cell nucleus. A dual-luciferase reporter assay confirmed that CsbHLH024 and CsbHLH133 served as activators with transcriptional activity in tea plant (Figure 6B). 

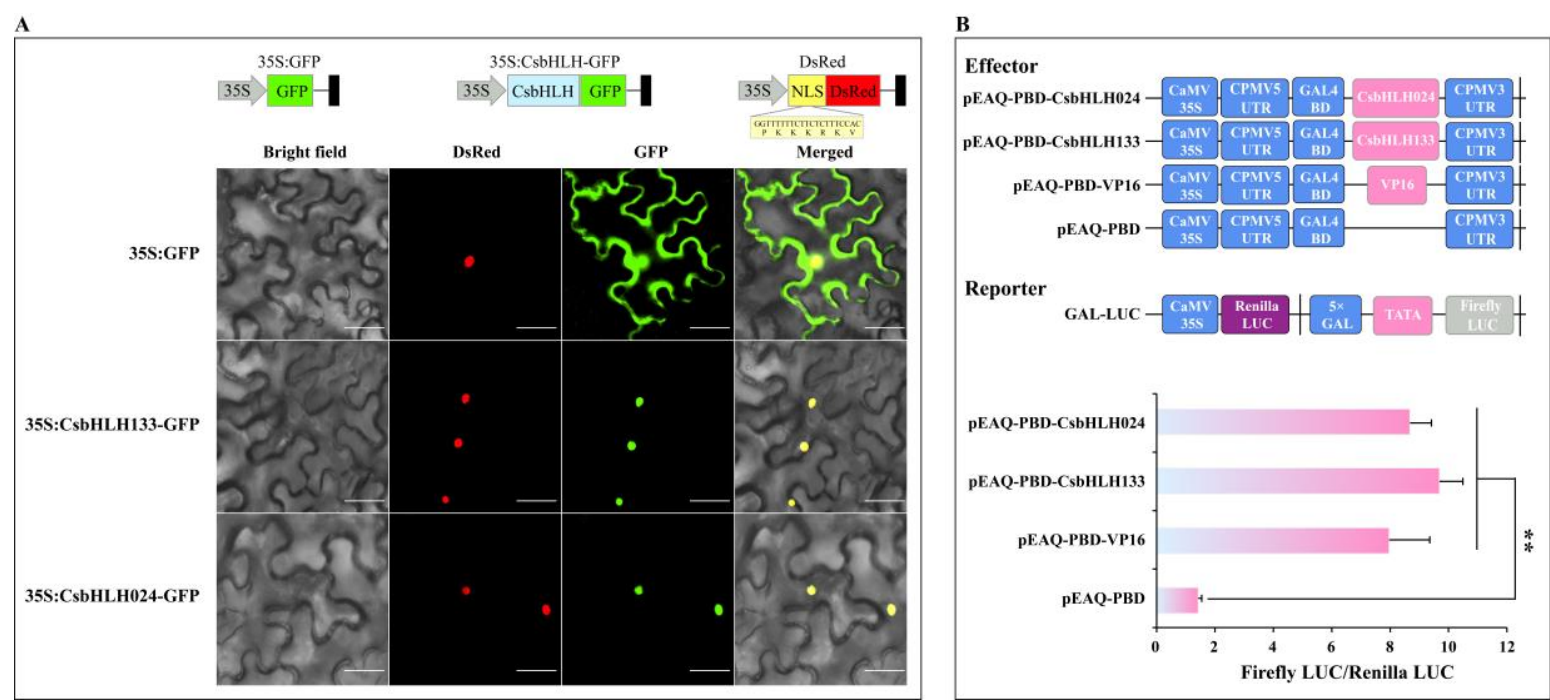

Figure 6. Transcriptional activity of CsbHLH133 and CsbHLH024 and the functional protein association network. A. The subcellular location of CsbHLH133 and CsbHLH024 in Nicotiana benthamiana. Scale bars $=50 \mu \mathrm{m}$. B. The transcriptional activation of CsbHLH133 and CsbHLH024 in tobacco. The data are presented as the means \pm SDs (n=7). The empty vector (PBD) and pBD-VP16 were used as negative and positive controls, respectively. Significant differences were determined using Student's t-test by comparison to the negative control $\left({ }^{* *}, \mathrm{P}<0.01\right)$.

\section{Potential protein regulatory network of trichome formation}

bHLH TFs usually exert functions by interacting with other TFs, therefore, protein interactions are important to predicate bHLH protein function. A potential functional protein association network in tea plant was constructed on the basis of the regulation of Arabidopsis thaliana trichome formation by multiple TFs, including MYB23, TRY, GL3, ETC1, GL1, GL2, EGL3, SAD2, ETC2, TTG1 and CPC [57] (Figure 7A). CsbHLH024 and CsbHLH133 likely regulate trichome formation by binding to multiple TFs. A yeast two-hybrid assay indicated that CsbHLH024 and CsbHLH133 could interact with a regulator of tea trichome formation, CsTTG1 (Figure 7B). In addition, the expression of CsTTG1 maintained a good agreement with that of CsbHLH024 and CsbHLH133 (Figure 7C). These results suggested that CsbHLH024 and CsbHLH133 might regulate trichome formation by interacting with multiple TFs, especially CsTTG1. 
A

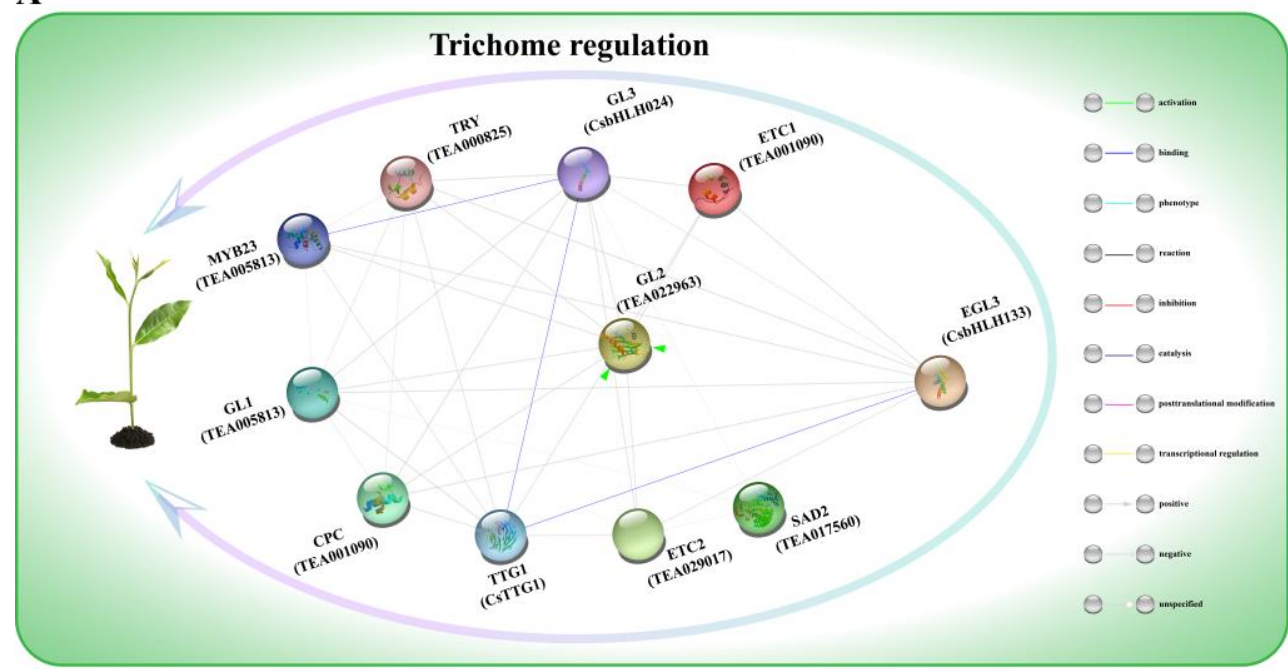

B

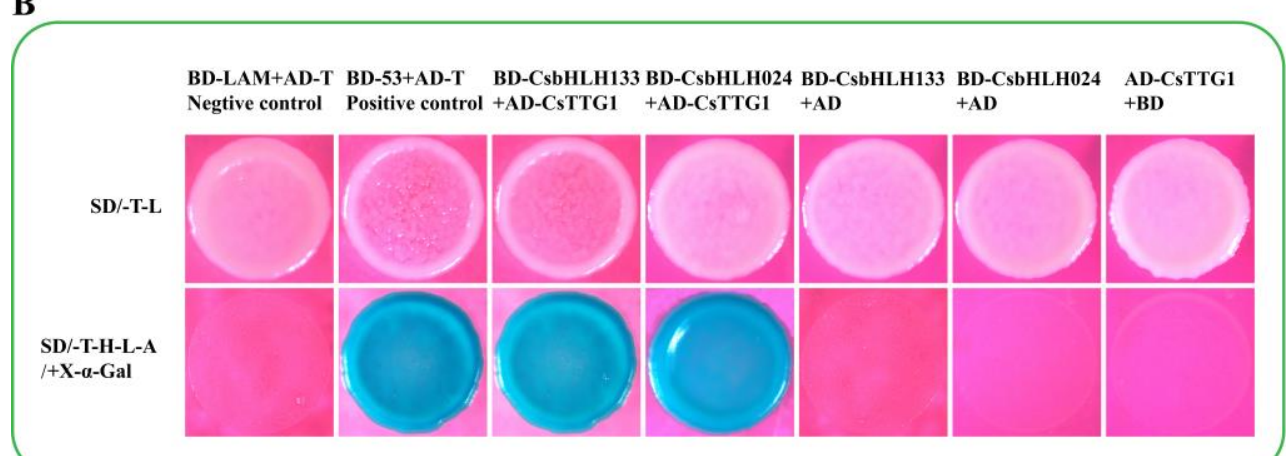

C

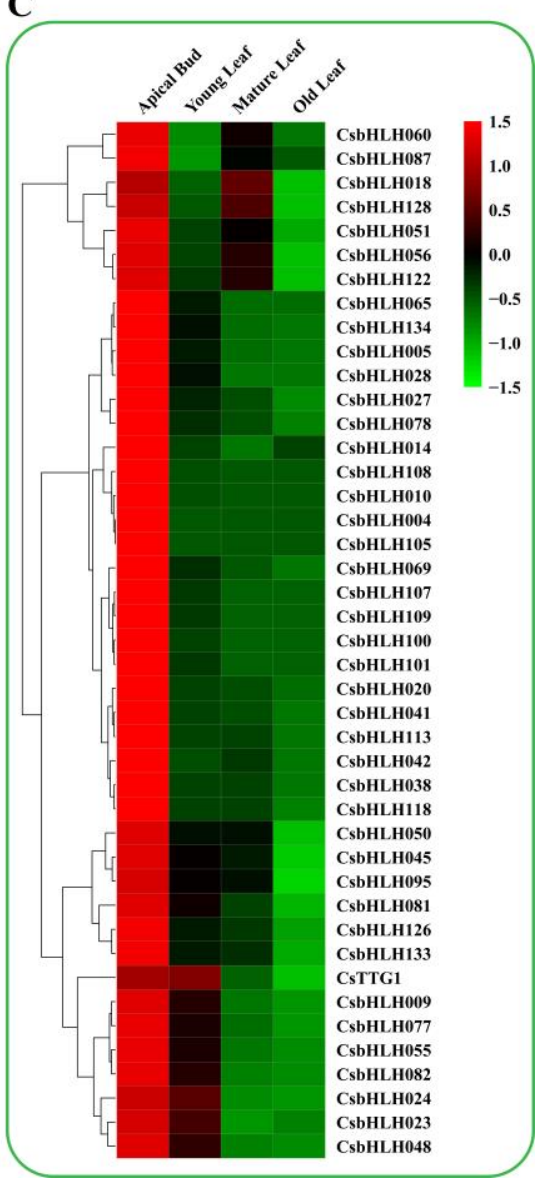

Figure 7. Functional protein association network of trichome formation and a yeast two-hybrid assay. A. The potential tea trichome regulatory network. A functional protein association network was constructed based on the TFs associated with Arabidopsis thaliana trichome formation. Homologous genes were found in the CSS genome, including CsbHLH024 and CsbHLH133. The name of homologous CsbHLH is shown in brackets under Arabidopsis thaliana. B. Yeast two-hybrid assay of protein-protein interactions between CsbHLH024 or CsbHLH133 and CsTTG1. BD and AD represent empty pGBKT7 and pGADT7 vectors, respectively. The SD/-T-L synthetic dextrose (SD) medium lacked tryptophan and leucine; the SD/-T-H-L-A medium lacked tryptophan, histidine, leucine and adenine. Positive bacteria were stained using X- $\alpha$-Gal. C. The expression patterns of CsTTG1 and the members of cluster 3 in different developing leaf tissues. The name of each gene is listed on the right side of the heatmap.

\section{Discussion}

The identification of TF family members, such as MYBs [63], ERFs [64] and WRKYs [65], has been widely performed in many plants and has contributed to identifying gene functions. Several TF families have already been identified and systematically investigated for their functions in growth and development processes in tea plant; these families include the HSP gene superfamily [66], the VQ gene family [67] and the GRF and GIF gene families [68]. Trichome formation was conducive not only to plant resistance but also to tea flavor and aroma [27]. Numerous studies have demonstrated that bHLH TFs contribute to trichome formation [40-45]. However, whether bHLH TFs are involved in tea trichome formation is still unknown. In this study, a total of 134 CsbHLH genes were identified in the CSS genome. The bHLH family members in many species, including Zea mays L. (208) [69], Solanum lycopersicum (159) [70], Brassica rapa pekinensis (230) [31], Arabidopsis thaliana (147) [29], Glycine max (Linn.) Merr. (319) [71], Malus pumila (188) [72], Oryza sativa L. (167) [73] and Solanum tuberosum L. (124) [74], were successfully characterized and comprehensively investigated. The results suggested that the numbers of bHLH family members in different species were distinct. Twenty-three amino acid residues were conserved in the conserved bHLH domain, with a consensus rate greater than $50 \%$ (Figure 1), as observed in the previous studies [33,75]. The region including Glu-13 and Arg-16 (according to Glu-9 and Arg-12 in our alignment) 
could bind to the E-box, while the region including His-9, Glu-13 and Arg-17 (according to His-5, Glu-9 and Arg-13 in our alignment) could recognize the G-box [34,35,76]. Additionally, Glu-13 and Arg-17 (according to Glu-9 and Arg-13 in our alignment) were important for DNA binding, and Leu-27 (according to Leu-23 in our alignment) played a vital role in dimerization activity [77,78]. Members of the same group exhibited similar gene structures and motifs (Figure S4). However, the functions of most motifs have not been identified in plants.

Members of the same group in the phylogenetic tree might possess similar functions. All CsbHLH proteins were divided into 19 subgroups according to the Arabidopsis thaliana classification [58]. ICE and ICE2 are related to the cold acclimation response and freezing tolerance in Arabidopsis thaliana [79,80]; they were mapped to AtbHLH116 and AtbHLH033 and located in group IIIa. CsbHLH001, identified as CsICE1, might be involved in the ICE1-CBF cold response pathway in tea plant [81] and was also classified into group IIIa. FIT was mapped to AtbHLH029 and included in group IIIa; this protein is responsive to iron deficiency in Arabidopsis thaliana roots [82]. It is likely that the members of group IIIa are involved in the response to abiotic stress. Group IIIc-I and group IIIc-II mainly contained the bHLHs of Arabidopsis thaliana, which were associated with trichome formation and were defined as 'trichome-related groups' (Figure 2). GL3 and EGL3 are involved in plant trichome formation [40,41,43,45]; they were respectively mapped to CsbHLH024 and CsbHLH133 respectively in this study and belonged to the 'trichome-related groups' (Figure 2). The expression of members of cluster 1, cluster 3 and cluster 5 was in agreement with tea trichome distribution (Figure 3). CsbHLH024 and CsbHLH133 were also divided into these clusters. Their expression peaked in the young tissues of different cultivars, including apical buds and young leaves (Figure 5B). Moreover, CsbHLH024 and CsbHLH133 possess transcriptional activity (Figure 6B), and a protein interaction network related to trichome formation was predicted (Figure 7A). The results indicated that they might be involved in trichome formation by interacting with other TFs [4244]. CsTTG1 is involved in trichome formation. Overexpression of CsTTG1 could enhance Arabidopsis thaliana trichome density [83]. The yeast two-hybrid assay results suggested that CsbHLH024 and CsbHLH133 can interact with CsTTG1 in vitro (Figure 7B). It is likely that CsbHLH024 and CsbHLH133 participated in the regulation of trichome formation by interacting with CsTTG1.

However, the regeneration rate of tea plant explants in vitro is low because tea plant is a perennial woody species. Tea plant tissues are rich in the polyphenols. Polyphenols can inhibit the activity of Agrobacterium tumefaciens, which results in a low efficiency of tea plant genetic transformation [84]. The stable genetic transformation system of tea plant is still unavailable and needs to continue exploration. Thus, the function of CsbHLH024 and CsbHLH133 in the regulation of trichome formation must be further addressed.

\section{Conclusions}

A total of $134 \mathrm{CsbHLH}$ proteins were identified in the tea plant genome. Phylogenetic tree, gene structure and protein motif analyses of these proteins were conducted in this study. All CsbHLH proteins were divided into 19 subgroups. The members of group IIIc-I and group IIIc-II were associated with trichome formation and were defined as the 'trichome-related group'. The candidate genes related to trichome formation in tea plant were primarily located in cluster 1, cluster 3 and cluster 5. The expression patterns of CsbHLH024 and CsbHLH133, which were found in cluster 3 and classified into the 'trichome-related group', were similar to the trichome distribution observed in tea plants, with the peak occurring in the young tissues of different cultivars. In addition, CsbHLH024 and CsbHLH133 showed transcriptional activation ability and were likely to control trichome formation by interacting with CsTTG1. This study provides useful information for the further study of CsbHLH TF function in the regulation of trichome formation in tea plant. 


\section{References:}

1. Schilmiller, A.L.; Last, R.L.; Pichersky, E. Harnessing plant trichome biochemistry for the production of useful compounds. PLANT J. 54, 702-711 (2008).

2. Werker, E. Trichome Diversity and Development. ADV BOT RES. 31, 1-35 (2000).

3. Serna, L.; Martin, C. Trichomes: different regulatory networks lead to convergent structures. TRENDS PLANT SCI. 11, 274-280 (2006).

4. Ishida, T.; Kurata, T.; Okada, K.; Wada, T. A genetic regulatory network in the development of trichomes and root hairs. ANNU REV PLANT BIOL. 59, 365-386 (2008).

5. Wagner, G.J.; Wang, E.; Shepherd, R.W. New approaches for studying and exploiting an old protuberance, the plant trichome. Ann Bot. 93, 3-11 (2004).

6. Glas, J.J.; Schimmel, B.C.; Alba, J.M.; Escobar-Bravo, R.; Schuurink, R.C.; Kant, M.R. Plant glandular trichomes as targets for breeding or engineering of resistance to herbivores. INT J MOL SCI. 13, 17077-17103 (2012).

7. Bergau, N.; Bennewitz, S.; Syrowatka, F.; Hause, G.; Tissier, A. The development of type VI glandular trichomes in the cultivated tomato Solanum lycopersicum and a related wild species S. habrochaites. BMC PLANT BIOL. 15, 289 (2015).

8. Yue, C.; Cao, H.L.; Chen, D.; Lin, H.Z.; Wang, Z.; Hu, J.; Yang, G.Y.; Guo, Y.Q.; Ye, N.X.; Hao, X.Y. Comparative transcriptome study of hairy and hairless tea plant (Camellia sinensis) shoots. J PLANT PHYSIOL. 229, 41-52 (2018).

9. Bleeker, P.M.; Mirabella, R.; Diergaarde, P.J.; VanDoorn, A.; Tissier, A.; Kant, M.R.; Prins, M.; de Vos, M.; Haring, M.A.; Schuurink, R.C., Improved herbivore resistance in cultivated tomato with the sesquiterpene biosynthetic pathway from a wild relative. Proc Natl Acad Sci U S A. 109, 20124-20129 (2012).

10. Luu, V.T.; Weinhold, A.; Ullah, C.; Dressel, S.; Schoettner, M.; Gase, K.; Gaquerel, E.; Xu, S.; Baldwin, I.T. O-Acyl Sugars Protect a Wild Tobacco from Both Native Fungal Pathogens and a Specialist Herbivore. PLANT PHYSIOL. 174, 370-386 (2017).

11. YAMASAKI, S.; MURAKAMI, Y. Continuous UV-B Irradiation Induces Endoreduplication and Trichome Formation in Cotyledons, and Reduces Epidermal Cell Division and Expansion in the First Leaves of Pumpkin Seedlings (Cucurbita maxima Duch.^^^^times;C. moschata Duch.). Environmental Control in Biology. 52, 203-209 (2014).

12. Agrawal, A.A.; Conner, J.K.; Stinchcombe, J.R. Evolution of plant resistance and tolerance to frost damage. ECOL LETT. 7, 11991208 (2004).

13. Benz, B.W.; Martin, C.E. Foliar trichomes, boundary layers, and gas exchange in 12 species of epiphytic Tillandsia (Bromeliaceae). J PLANT PHYSIOL. 163, 648-656 (2013).

14. Skaltsa, H.; Verykokidou, E.; Harvala, C.; Karabourniotis, G.; Manetasi, Y. UV-B protective potential and flavonoid content of leaf hairs of Quercus ilex. PHYTOCHEMISTRY. 37, 987-990 (1994).

15. Chang, J.; Yu, T.; Yang, Q.; Li, C.; Xiong, C.; Gao, S.; Xie, Q.; Zheng, F.; Li, H.; Tian, Z.; Yang, C.; Ye, Z. Hair, encoding a single $\mathrm{C} 2 \mathrm{H} 2$ zinc-finger protein, regulates multicellular trichome formation in tomato. PLANT J. 96, 90-102 (2018).

16. Sun, W.; Gao, D.; Xiong, Y.; Tang, X.; Xiao, X.; Wang, C.; Yu, S. Hairy Leaf 6, an AP2/ERF Transcription Factor, Interacts with OsWOX3B and Regulates Trichome Formation in Rice. MOL PLANT. 10, 1417-1433 (2017).

17. Zhang, N.; Yang, L.; Luo, S.; Wang, X.; Wang, W.; Cheng, Y.; Tian, H.; Zheng, K.; Cai, L.; Wang, S. Genetic evidence suggests that GIS functions downstream of TCL1 to regulate trichome formation in Arabidopsis. BMC PLANT BIOL. 18, 63 (2018).

18. Liu, Y.; Liu, D.; Khan, A.R.; Liu, B.; Wu, M.; Huang, L.; Wu, J.; Song, G.; Ni, H.; Ying, H.; Yu, H.; Gan, Y. NbGIS regulates glandular trichome initiation through GA signaling in tobacco. PLANT MOL BIOL. 98, 153-167 (2018).

19. Yang, S.; Cai, Y.; Liu, X.; Dong, M.; Zhang, Y.; Chen, S.; Zhang, W.; Li, Y.; Tang, M.; Zhai, X.; Weng, Y.; Ren, H. A CsMYB6CsTRY module regulates fruit trichome initiation in cucumber. J EXP BOT. 69, 1887-1902 (2018).

20. Ma, D.; Hu, Y.; Yang, C.; Liu, B.; Fang, L.; Wan, Q.; Liang, W.; Mei, G.; Wang, L.; Wang, H.; Ding, L.; Dong, C.; Pan, M.; Chen, J.; Wang, S.; Chen, S.; Cai, C.; Zhu, X.; Guan, X.; Zhou, B.; Zhu, S.; Wang, J.; Guo, W.; Chen, X.; Zhang, T. Genetic basis for glandular trichome formation in cotton. NAT COMMUN. 7, 10456 (2016). 
21. Liu, S.; Fan, L.; Liu, Z.; Yang, X.; Zhang, Z.; Duan, Z.; Liang, Q.; Imran, M.; Zhang, M.; Tian, Z. A Pd1-Ps-P1 Feedback Loop Controls Pubescence Density in Soybean. MOL PLANT. S1674-S2052, 30350-30356 (2020).

22. Marks, M.D. Molecular genetic analysis of trichome development in Arabidopsis. Annu Rev Plant Physiol Plant Mol Biol. 48, 137163 (1997).

23. Xia, E.H.; Zhang, H.B.; Sheng, J.; Li, K.; Zhang, Q.J.; Kim, C.; Zhang, Y.; Liu, Y.; Zhu, T.; Li, W.; Huang, H.; Tong, Y.; Nan, H.; Shi, C.; Shi, C.; Jiang, J.J.; Mao, S.Y.; Jiao, J.Y.; Zhang, D.; Zhao, Y.; Zhao, Y.J.; Zhang, L.P.; Liu, Y.L.; Liu, B.Y.; Yu, Y.; Shao, S.F.; Ni, D.J.; Eichler, E.E.; Gao, L.Z. The Tea Tree Genome Provides Insights into Tea Flavor and Independent Evolution of Caffeine Biosynthesis. MOL PLANT. 10, 866-877 (2017).

24. Gong, Y.; Zhang, Q.; Lin, Y.; Zeng, X.; Wang, X.; Luo, F.; Liu, S.; Gao, L.; Cai, S.; Chen, Z.; Xiong, L.; Zhang, D.; Zhang, S.; Huang, J.; Li, W.; Li, Q.; Liu, Z.; Zhou, L. Leading progress on genomics, health benefits and utilization of tea resources in China. NATURE. 566, S15-S19 (2019).

25. Gilbert, N. The science of tea's mood-altering magic. NATURE. 566, S8-S9 (2019).

26. Wei, C.; Yang, H.; Wang, S.; Zhao, J.; Liu, C.; Gao, L.; Xia, E.; Lu, Y.; Tai, Y.; She, G.; Sun, J.; Cao, H.; Tong, W.; Gao, Q.; Li, Y.; Deng, W.; Jiang, X.; Wang, W.; Chen, Q.; Zhang, S.; Li, H.; Wu, J.; Wang, P.; Li, P.; Shi, C.; Zheng, F.; Jian, J.; Huang, B.; Shan, D.; Shi, M.; Fang, C.; Yue, Y.; Li, F.; Li, D.; Wei, S.; Han, B.; Jiang, C.; Yin, Y.; Xia, T.; Zhang, Z.; Bennetzen, J.L.; Zhao, S.; Wan, $X$. Draft genome sequence of Camellia sinensis var. sinensis provides insights into the evolution of the tea genome and tea quality. Proc Natl Acad Sci U S A. 115, E4151-E4158 (2018).

27. Li, P.; Xu, Y.; Zhang, Y.; Fu, J.; Yu, S.; Guo, H.; Chen, Z.; Chen, C.; Yang, X.; Wang, S.; Zhao, J. Metabolite Profiling and Transcriptome Analysis Revealed the Chemical Contributions of Tea Trichomes to Tea Flavors and Tea Plant Defenses. J Agric Food Chem. 68, 11389-11401 (2020).

28. Feller, A.; Machemer, K.; Braun, E.L.; Grotewold, E. Evolutionary and comparative analysis of MYB and bHLH plant transcription factors. PLANT J. 66, 94-116 (2011).

29. Toledo-Ortiz, G.; Huq, E.; Quail, P.H. The Arabidopsis basic/helix-loop-helix transcription factor family. PLANT CELL. 15, 17491770 (2003).

30. Yin, J.; Chang, X.; Kasuga, T.; Bui, M.; Reid, M.S.; Jiang, C.Z. A basic helix-loop-helix transcription factor, PhFBH4, regulates flower senescence by modulating ethylene biosynthesis pathway in petunia. Hortic Res. 2, 15059 (2015).

31. Song, X.M.; Huang, Z.N.; Duan, W.K.; Ren, J.; Liu, T.K.; Li, Y.; Hou, X.L. Genome-wide analysis of the bHLH transcription factor family in Chinese cabbage (Brassica rapa ssp. pekinensis). MOL GENET GENOMICS. 289, 77-91 (2014).

32. Murre, C.; McCaw, P.S.; Baltimore, D. A new DNA binding and dimerization motif in immunoglobulin enhancer binding, daughterless, MyoD, and myc proteins. CELL. 56, 777-783 (1989).

33. Atchley, W.R.; Terhalle, W.; Dress, A. Positional dependence, cliques, and predictive motifs in the bHLH protein domain. JMOL EVOL. 48, 501-516 (1999).

34. Massari, M.E.; Murre, C. Helix-loop-helix proteins: regulators of transcription in eucaryotic organisms. MOL CELL BIOL. 20, 429-440 (2000).

35. Ferre-D'Amare, A.R.; Pognonec, P.; Roeder, R.G.; Burley, S.K. Structure and function of the b/HLH/Z domain of USF. EMBO J. 13, 180-189 (1994).

36. Ledent, V.; Vervoort, M. The basic helix-loop-helix protein family: comparative genomics and phylogenetic analysis. GENOME RES. 11, 754-770 (2001).

37. Sun, X.; Wang, Y.; Sui, N. Transcriptional regulation of bHLH during plant response to stress. Biochem Biophys Res Commun. 503, 397-401 (2018).

38. Mertens, J.; Pollier, J.; Vanden, B.R.; Lopez-Vidriero, I.; Franco-Zorrilla, J.M.; Goossens, A. The bHLH Transcription Factors TSAR1 and TSAR2 Regulate Triterpene Saponin Biosynthesis in Medicago truncatula. PLANT PHYSIOL. 170, 194-210 (2016). 
39. Sun, B.; Zhu, Z.; Cao, P.; Chen, H.; Chen, C.; Zhou, X.; Mao, Y.; Lei, J.; Jiang, Y.; Meng, W.; Wang, Y.; Liu, S. Purple foliage coloration in tea (Camellia sinensis L.) arises from activation of the R2R3-MYB transcription factor CsAN1. Sci Rep. 6, 32534 (2016).

40. Payne, C.T.; Zhang, F.; Lloyd, A.M. GL3 encodes a bHLH protein that regulates trichome development in Arabidopsis through interaction with GL1 and TTG1. GENETICS. 156, 1349-1362 (2000).

41. Zhang, F.; Gonzalez, A.; Zhao, M.; Payne, C.T.; Lloyd, A. A network of redundant bHLH proteins functions in all TTG1dependent pathways of Arabidopsis. DEVELOPMENT. 130, 4859-4869 (2003).

42. Rerie, W.G.; Feldmann, K.A.; Marks, M.D. The GLABRA2 gene encodes a homeo domain protein required for normal trichome development in Arabidopsis. Genes Dev. 8, 1388-1399 (1994).

43. Zhao, M.; Morohashi, K.; Hatlestad, G.; Grotewold, E.; Lloyd, A. The TTG1-bHLH-MYB complex controls trichome cell fate and patterning through direct targeting of regulatory loci. DEVELOPMENT. 135, 1991-1999 (2008).

44. Balkunde, R.; Pesch, M.; Hulskamp, M. Trichome patterning in Arabidopsis thaliana from genetic to molecular models. CURR TOP DEV BIOL. 91, 299-321 (2010).

45. Luo, D.; Oppenheimer, D.G. Genetic control of trichome branch number in Arabidopsis: the roles of the FURCA loci. DEVELOPMENT. 126, 5547-5557 (1999).

46. Tominaga-Wada, R.; Nukumizu, Y.; Sato, S.; Wada, T. Control of plant trichome and root-hair development by a tomato (Solanum lycopersicum) R3 MYB transcription factor. PLOS ONE. 8, e54019 (2013).

47. Letunic, I.; Bork, P. 20 years of the SMART protein domain annotation resource. NUCLEIC ACIDS RES. 46, D493-D496 (2018).

48. Lu, S.; Wang, J.; Chitsaz, F.; Derbyshire, M.K.; Geer, R.C.; Gonzales, N.R.; Gwadz, M.; Hurwitz, D.I.; Marchler, G.H.; Song, J.S.; Thanki, N.; Yamashita, R.A.; Yang, M.; Zhang, D.; Zheng, C.; Lanczycki, C.J.; Marchler-Bauer, A. CDD/SPARCLE: the conserved domain database in 2020. NUCLEIC ACIDS RES. 48, D265-D268 (2020).

49. Finn, R.D.; Coggill, P.; Eberhardt, R.Y.; Eddy, S.R.; Mistry, J.; Mitchell, A.L.; Potter, S.C.; Punta, M.; Qureshi, M.; SangradorVegas, A.; Salazar, G.A.; Tate, J.; Bateman, A. The Pfam protein family database: towards a more sustainable future. NUCLEIC ACIDS RES. 44, D279-D285 (2016).

50. Bailey, T.L.; Boden, M.; Buske, F.A.; Frith, M.; Grant, C.E.; Clementi, L.; Ren, J.; Li, W.W.; Noble, W.S. MEME SUITE: tools for motif discovery and searching. NUCLEIC ACIDS RES. 37, (Web Server issue), W202-W208 (2009).

51. Chen, C.; Chen, H.; Zhang, Y.; Thomas, H.R.; Frank, M.H.; He, Y.; Xia, R. TBtools: An Integrative Toolkit Developed for Interactive Analyses of Big Biological Data. MOL PLANT. 13, 1194-1202 (2020).

52. Tian, F.; Yang, D.C.; Meng, Y.Q.; Jin, J.; Gao, G. PlantRegMap: charting functional regulatory maps in plants. NUCLEIC ACIDS RES. 48, D1104-D1113 (2020).

53. Kumar, S.; Stecher, G.; Li, M.; Knyaz, C.; Tamura, K. MEGA X: Molecular Evolutionary Genetics Analysis across Computing Platforms. MOL BIOL EVOL. 35, 1547-1549 (2018).

54. Subramanian, B.; Gao, S.; Lercher, M.J.; Hu, S.; Chen, W.H. Evolview v3: a webserver for visualization, annotation, and management of phylogenetic trees. NUCLEIC ACIDS RES. 47, W270-W275 (2019).

55. Zhu, Z.; Sun, B.; Cai, W.; Zhou, X.; Mao, Y.; Chen, C.; Wei, J.; Cao, B.; Chen, C.; Chen, G.; Lei, J. Natural variations in the MYB transcription factor MYB31 determine the evolution of extremely pungent peppers. NEW PHYTOL. 223, 922-938 (2019).

56. Livak, K.J.; Schmittgen, T.D. Analysis of relative gene expression data using real-time quantitative PCR and the 2(-Delta Delta C(T)) Method. METHODS. 25, 402-408 (2001).

57. Schellmann, S.; Hulskamp, M. Epidermal differentiation: trichomes in Arabidopsis as a model system. INT J DEV BIOL. 49, 579584 (2005).

58. Heim, M.A.; Jakoby, M.; Werber, M.; Martin, C.; Weisshaar, B.; Bailey, P.C. The basic helix-loop-helix transcription factor family in plants: a genome-wide study of protein structure and functional diversity. MOL BIOL EVOL. 20, 735-747 (2003).

59. Nesi, N.; Debeaujon, I.; Jond, C.; Pelletier, G.; Caboche, M.; Lepiniec, L. The TT8 gene encodes a basic helix-loop-helix domain protein required for expression of DFR and BAN genes in Arabidopsis siliques. PLANT CELL. 12, 1863-1878 (2000). 
60. Bernhardt, C.; Lee, M.M.; Gonzalez, A.; Zhang, F.; Lloyd, A.; Schiefelbein, J. The bHLH genes GLABRA3 (GL3) and ENHANCER OF GLABRA3 (EGL3) specify epidermal cell fate in the Arabidopsis root. DEVELOPMENT. 130, 6431-6439 (2003).

61. Morohashi K.; Zhao M.; Yang M.; Read B.; Lloyd A.; Lamb R.; Grotewold E. Participation of the Arabidopsis bHLH factor GL3 in trichome initiation regulatory events. Plant Physiol. 145, 736-746 (2007).

62. Pesch, M.; Schultheiss, I.; Digiuni, S.; Uhrig, J.F.; Hulskamp, M. Mutual control of intracellular localisation of the patterning proteins AtMYC1, GL1 and TRY/CPC in Arabidopsis. DEVELOPMENT. 140, 3456-3467 (2013).

63. Peng, X.; Liu, H.; Wang, D.; Shen, S. Genome-wide identification of the Jatropha curcas MYB family and functional analysis of the abiotic stress responsive gene JcMYB2. BMC GENOMICS. 17, 251 (2016).

64. Song, J.; Chen, C.; Zhang, S.; Wang, J.; Huang, Z.; Chen, M.; Cao, B.; Zhu, Z.; Lei, J. Systematic analysis of the Capsicum ERF transcription factor family: identification of regulatory factors involved in the regulation of species-specific metabolites. $B M C$ GENOMICS. 21, 573 (2020).

65. Jue, D.; Sang, X.; Liu, L.; Shu, B.; Wang, Y.; Liu, C.; Xie, J.; Shi, S. Identification of WRKY Gene Family from Dimocarpus longan and Its Expression Analysis during Flower Induction and Abiotic Stress Responses. INT J MOL SCI. 19, 2169 (2018).

66. Chen, J.; Gao, T.; Wan, S.; Zhang, Y.; Yang, J.; Yu, Y.; Wang, W. Genome-Wide Identification, Classification and Expression Analysis of the HSP Gene Superfamily in Tea Plant (Camellia sinensis). INT J MOL SCI. 19, 2633 (2018).

67. Guo, J.; Chen, J.; Yang, J.; Yu, Y.; Yang, Y.; Wang, W. Identification, characterization and expression analysis of the VQ motifcontaining gene family in tea plant (Camellia sinensis). BMC GENOMICS. 19, 710 (2018).

68. Wu, Z.J.; Wang, W.L.; Zhuang, J. Developmental processes and responses to hormonal stimuli in tea plant (Camellia sinensis) leaves are controlled by GRF and GIF gene families. Funct Integr Genomics. 17, 503-512 (2017).

69. Zhang, T.; Lv, W.; Zhang, H.; Ma, L.; Li, P.; Ge, L.; Li, G. Genome-wide analysis of the basic Helix-Loop-Helix (bHLH) transcription factor family in maize. BMC PLANT BIOL. 18, 235 (2018).

70. Sun, H.; Fan, H.J.; Ling, H.Q. Genome-wide identification and characterization of the bHLH gene family in tomato. BMC GENOMICS. 16, 9 (2015).

71. Hudson, K.A.; Hudson, M.E. A classification of basic helix-loop-helix transcription factors of soybean. INT J GENOMICS. 2015, 603182 (2015).

72. Mao, K.; Dong, Q.; Li, C.; Liu, C.; Ma, F. Genome Wide Identification and Characterization of Apple bHLH Transcription Factors and Expression Analysis in Response to Drought and Salt Stress. FRONT PLANT SCI. 8, 480 (2017).

73. Li, X.; Duan, X.; Jiang, H.; Sun, Y.; Tang, Y.; Yuan, Z.; Guo, J.; Liang, W.; Chen, L.; Yin, J.; Ma, H.; Wang, J.; Zhang, D. Genomewide analysis of basic/helix-loop-helix transcription factor family in rice and Arabidopsis. PLANT PHYSIOL. 141, 1167-1184 (2006).

74. Wang, R.; Zhao, P.; Kong, N.; Lu, R.; Pei, Y.; Huang, C.; Ma, H.; Chen, Q. Genome-Wide Identification and Characterization of the Potato bHLH Transcription Factor Family. Genes (Basel). 9, 54 (2018).

75. Cui, X.; Wang, Y.X.; Liu, Z.W.; Wang, W.L.; Li, H.; Zhuang, J. Transcriptome-wide identification and expression profile analysis of the bHLH family genes in Camellia sinensis. Funct Integr Genomics. 18, 489-503 (2018).

76. Shimizu, T.; Toumoto, A.; Ihara, K.; Shimizu, M.; Kyogoku, Y.; Ogawa, N.; Oshima, Y.; Hakoshima, T. Cry stal structure of PHO4 bHLH domain-DNA complex: flanking base recognition. EMBO J. 16, 4689-4697 (1997).

77. Atchley, W.R.; Fitch, W.M. A natural classification of the basic helix-loop-helix class of transcription factors. Proc Natl Acad Sci U S A. 94, 5172-5176 (1997).

78. Carretero-Paulet, L.; Galstyan, A.; Roig-Villanova, I.; Martinez-Garcia, J.F.; Bilbao-Castro, J.R.; Robertson, D.L. Genome-wide classification and evolutionary analysis of the bHLH family of transcription factors in Arabidopsis, poplar, rice, moss, and algae. PLANT PHYSIOL. 153, 1398-1412 (2010).

79. Chinnusamy, V.; Ohta, M.; Kanrar, S.; Lee, B.H.; Hong, X.; Agarwal, M.; Zhu, J.K. ICE1: a regulator of cold-induced transcriptome and freezing tolerance in Arabidopsis. Genes Dev. 17, 1043-1054 (2003). 
80. Fursova, O.V.; Pogorelko, G.V.; Tarasov, V.A. Identification of ICE2, a gene involved in cold acclimation which determines freezing tolerance in Arabidopsis thaliana. GENE. 429, 98-103 (2009).

81. Wang, Y.; Jiang, C.J.; Li, Y.Y.; Wei, C.L.; Deng, W.W. CsICE1 and CsCBF1: two transcription factors involved in cold responses in Camellia sinensis. PLANT CELL REP. 31, 27-34 (2012).

82. Sivitz, A.; Grinvalds, C.; Barberon, M.; Curie, C.; Vert, G. Proteasome-mediated turnover of the transcriptional activator FIT is required for plant iron-deficiency responses. PLANT J. 66, 1044-1052 (2011).

83. Sun B.; Zhu Z., Liu R.; Wang L.; Dai F.; Cao F.; Liu S. TRANSPARENT TESTA GLABRA1 (TTG1) regulates leaf trichome density in tea Camellia sinensis. NORD J BOT. 38, (2020).

84. Mondal, T.K.; Bhattacharya, A.; Laxmikumaran, M.; Singh Ahuja, P. Recent Advances of Tea (Camellia Sinensis) Biotechnology. Plant Cell, Tissue and Organ Culture. 76, 195-254 (2004).

Author Contributions: RJL performed most experiments and wrote the manuscript. YYW analyzed the data. ST and JRC provided some useful suggestions regarding the study. PZ, BMS and SQL supervised the research.

490

491

492

493

494

495

496

497

498

499

500

501

502

503

504

Abbreviations

TFs

Chr

FPKM

bHLH

HLH

MEME

HMM

CSS

TAIR

MBW transcription factors

fragments per kilobase of transcript per million mapped reads

basic helix-loop-helix

helix-loop-helix

multiple em for motif elicitation

Camellia sinensis var. sinenesis

The Arabidopsis Information Resource

MYB-bHLH-WD40 chromosome

hidden markov model 
Figures

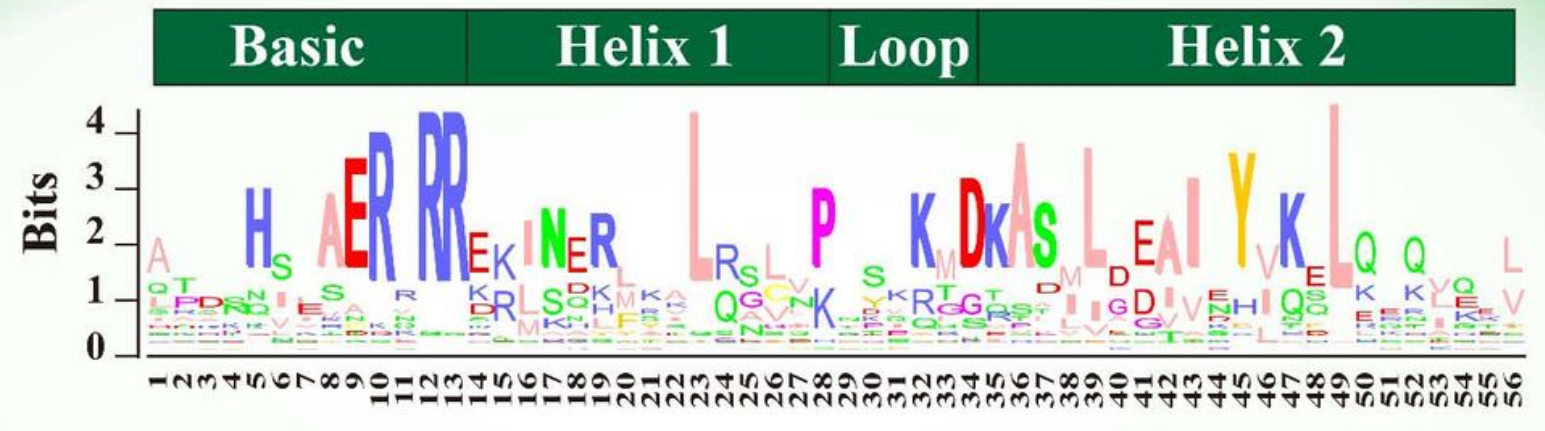

\begin{tabular}{|c|c|c|c|c|c|c|c|c|c|c|c|}
\hline Conserved aa $>50 \%$ & $\mathbf{H}$ & AER & $\mathbf{R} \mathbf{R}$ & $\mathbf{N}$ & $\mathbf{R}$ & $\mathbf{L}$ & $\mathbf{P}$ & K & DKAS & $\mathbf{L}$ & 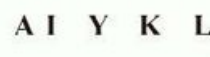 \\
\hline ons & $\frac{1}{6}$ & 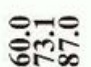 & సׁุ & ñ & $\bar{n}$ & $\stackrel{\circ}{\circ}$ & î & 8 & 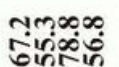 & 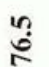 & 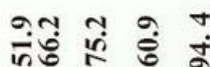 \\
\hline
\end{tabular}

Figure 1

Conserved residues analysis of bHLH domains. The height of each residue indicates the conservation rate. The black letters represent the residues with a consensus ratio greater than $50 \%$. 


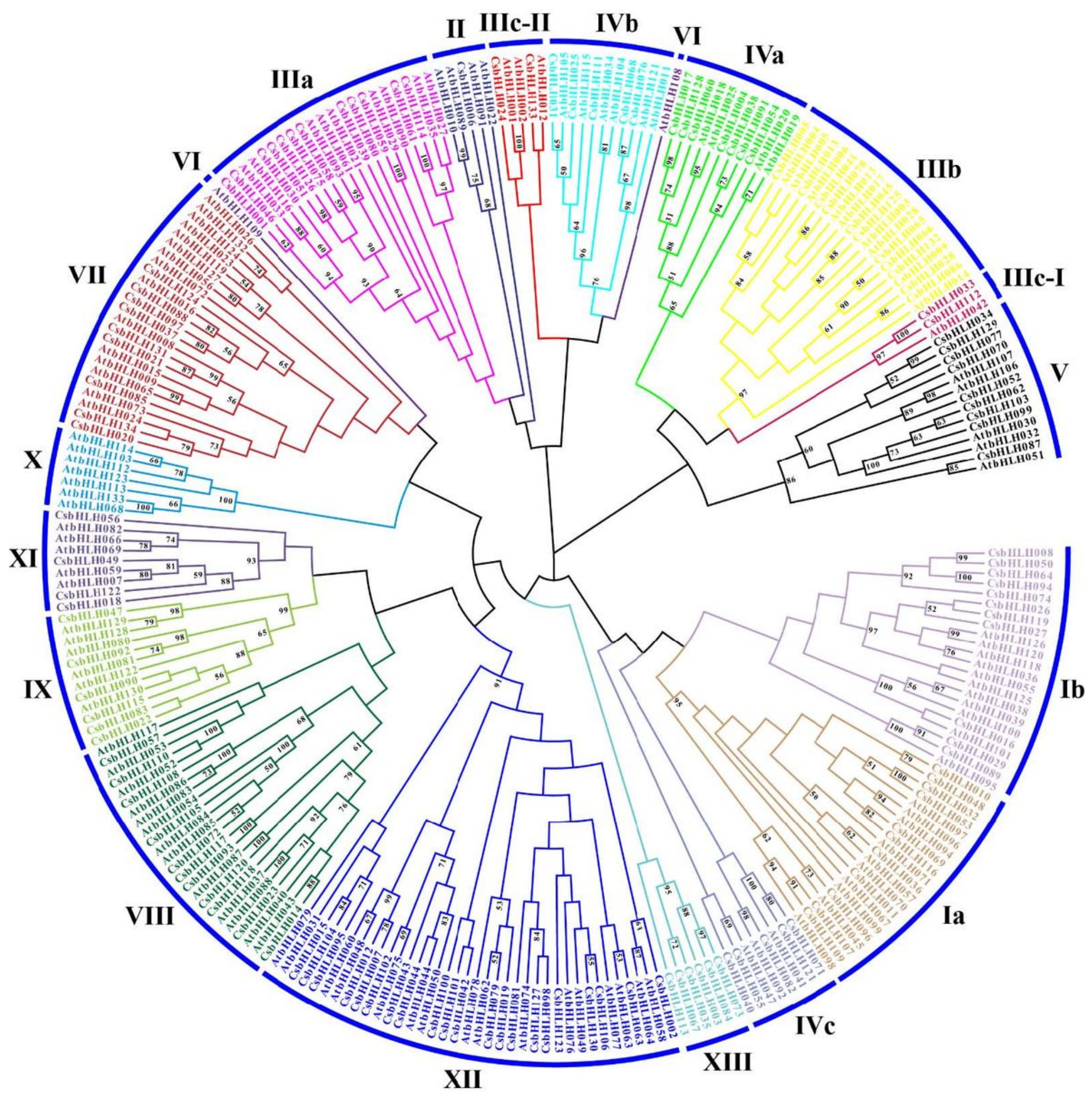

Figure 2

Phylogenetic tree analysis of tea plant and Arabidopsis thaliana bHLH proteins. Different colored branches indicate different subgroups. 


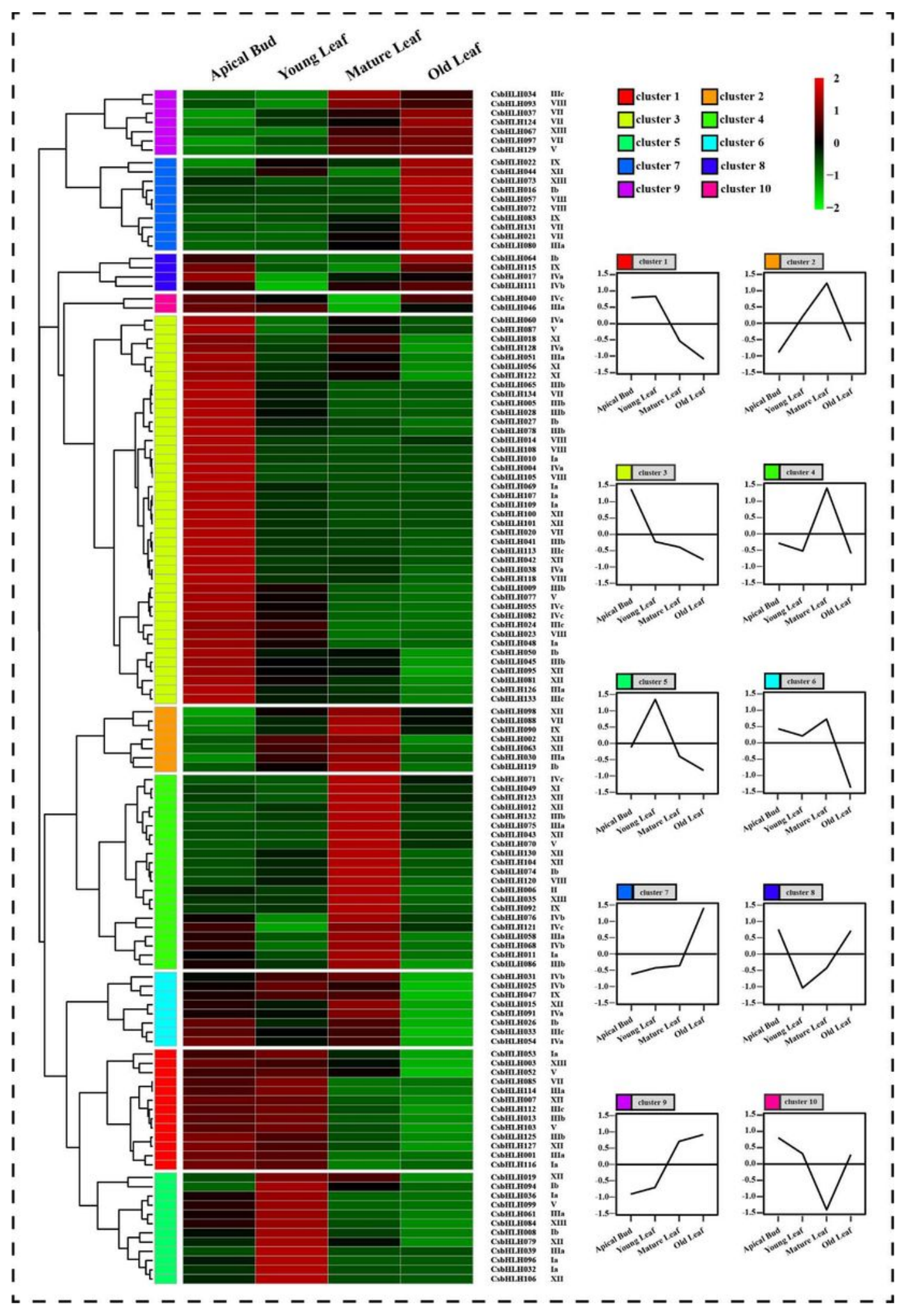

Figure 3

Transcriptome analysis of CsbHLHs in different developing leaf tissues. The name of each gene and the short name of the phylogenetic group are listed on the right of the heatmap. Line charts were generated using the mean value for the whole cluster. 


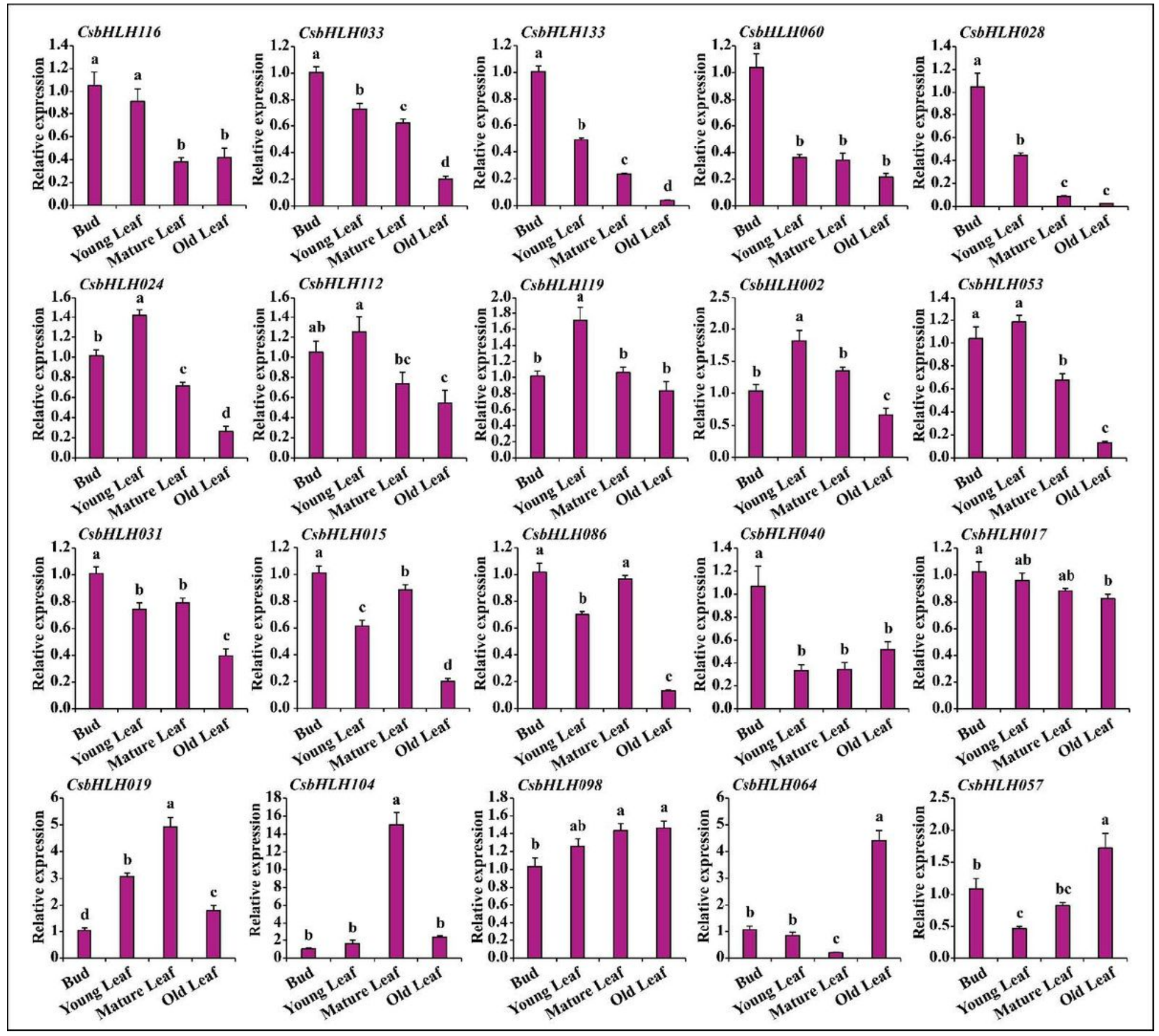

Figure 4

The expression patterns of CsbHLHs in different developing leaf tissues. The different letters in the figures indicate significantly different values ( $P<0.05$, Tukey's test). For all developing leaf tissues, three biological replicates and three technical replicates of each biological replicate were performed. 


RHBH

Figure 5

The trichome distribution and the expression patterns of CsbHLH024 and CsbHLH133 in different tea plant cultivars. A. The trichome distribution in cultivars 'RHBH', 'YH9' and 'BYDC'. B. The expression patterns of CsbHLH024 and CsbHLH133 in different developing leaf tissues of different tea plant cultivars. The different letters in the figures indicate significantly different values $(P<0.05$, Tukey's test).
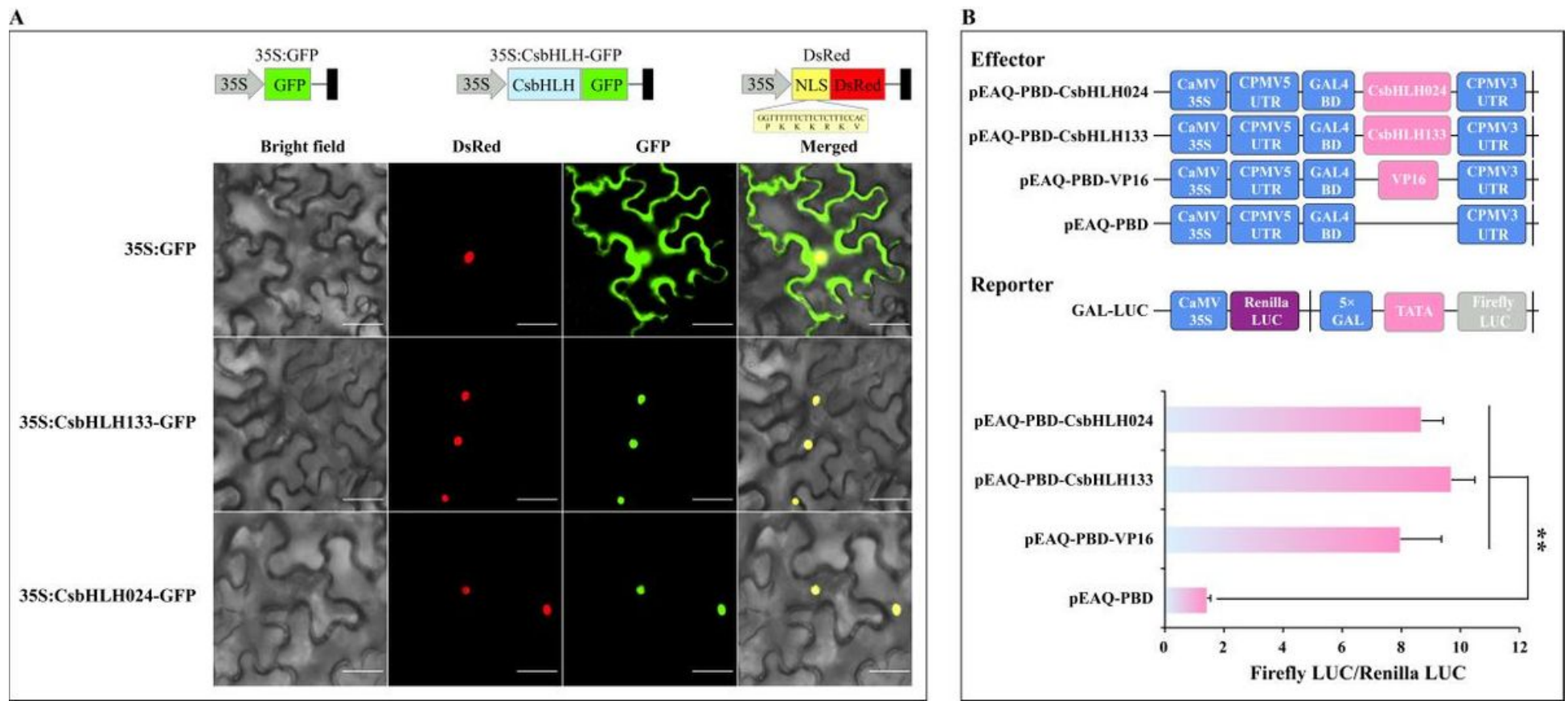

Figure 6 
Transcriptional activity of CsbHLH133 and CsbHLH024 and the functional protein association network. A. The subcellular location of CsbHLH133 and CsbHLH024 in Nicotiana benthamiana. Scale bars = 50 $\mu \mathrm{m}$. B. The transcriptional activation of CsbHLH133 and CsbHLH024 in tobacco. The data are presented as the means \pm SDs $(n=7)$. The empty vector (PBD) and pBD-VP16 were used as negative and positive controls, respectively. Significant differences were determined using Student's t-test by comparison to the negative control (**, $\mathrm{P}<0.01)$.

A

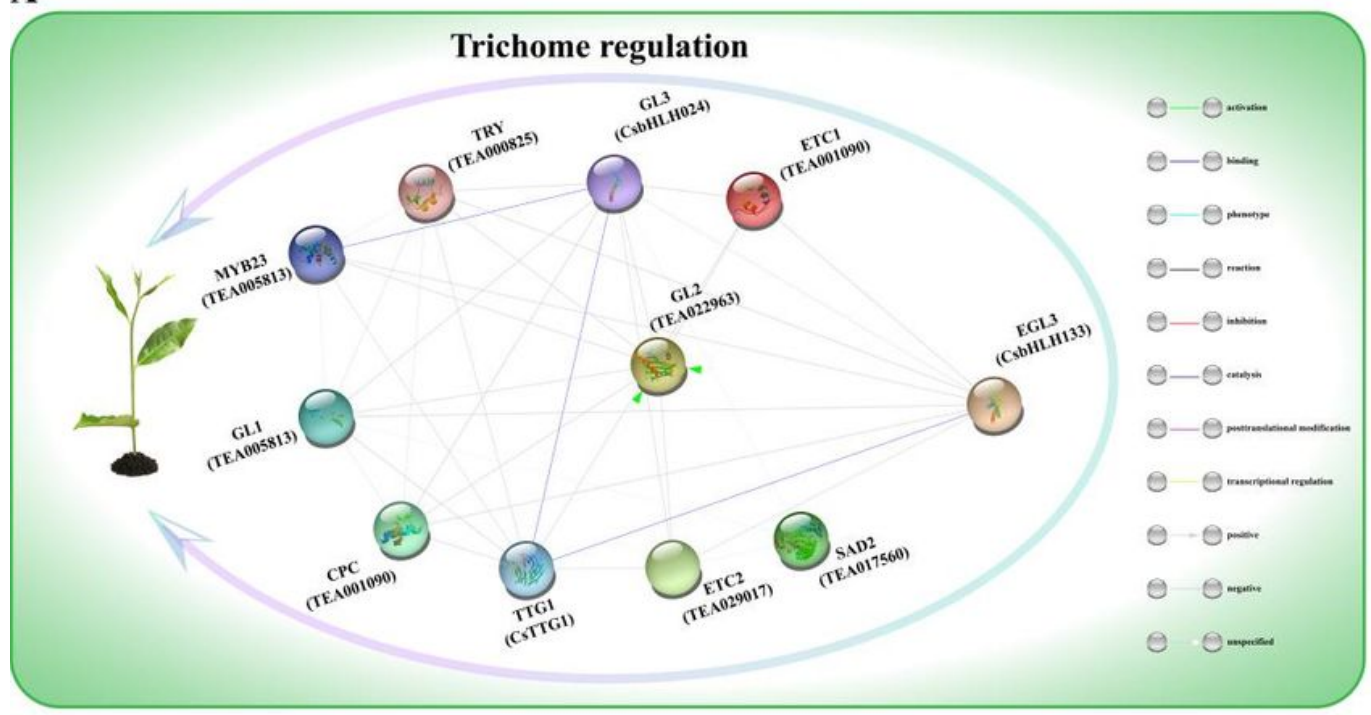

B

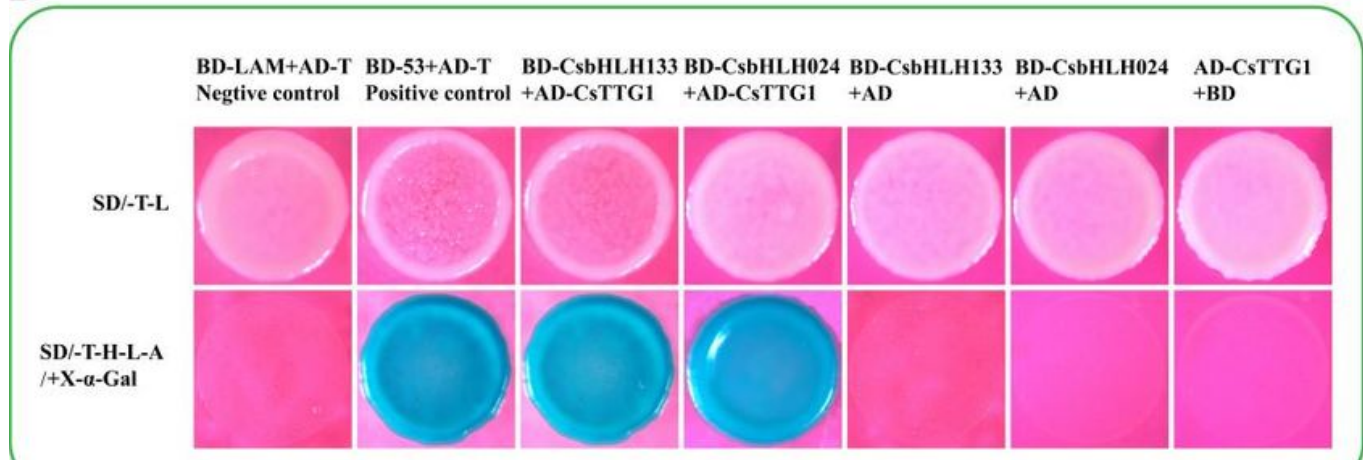

C

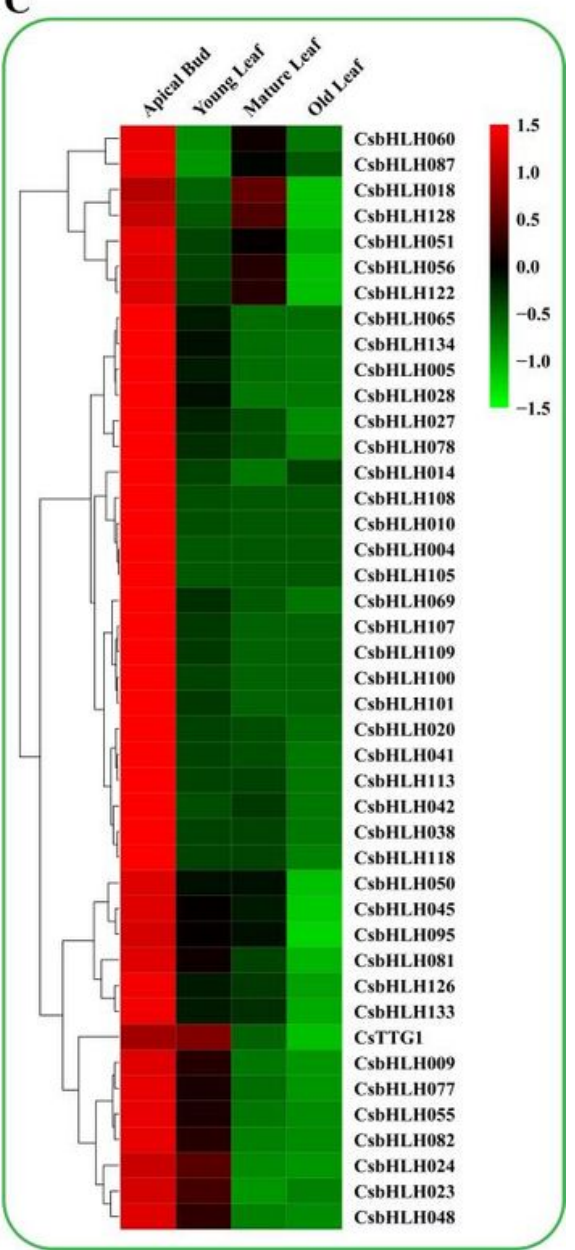

Figure 7

Functional protein association network of trichome formation and a yeast two-hybrid assay. A. The potential tea trichome regulatory network. A functional protein association network was constructed based on the TFs associated with Arabidopsis thaliana trichome formation. Homologous genes were found in the CSS genome, including CsbHLH024 and CsbHLH133. The name of homologous CsbHLH is shown in brackets under Arabidopsis thaliana. B. Yeast two-hybrid assay of protein-protein interactions between CsbHLH024 or CsbHLH133 and CsTTG1. BD and AD represent empty pGBKT7 and pGADT7 vectors, respectively. The SD/-T-L synthetic dextrose (SD) medium lacked tryptophan and leucine; the $\mathrm{SD} /-\mathrm{T}-\mathrm{H}-\mathrm{L}-\mathrm{A}$ medium lacked tryptophan, histidine, leucine and adenine. Positive bacteria were stained using X-a-Gal. C. The expression patterns of CsTTG1 and the members of cluster 3 in different developing leaf tissues. The name of each gene is listed on the right side of the heatmap. 


\section{Supplementary Files}

This is a list of supplementary files associated with this preprint. Click to download.

- Supplementaryfiles.zip 